This item was submitted to Loughborough's Research Repository by the author.

Items in Figshare are protected by copyright, with all rights reserved, unless otherwise indicated.

\title{
Dynamic analysis of multi-cracked Euler-Bernoulli beams with gradient elasticity
}

PLEASE CITE THE PUBLISHED VERSION

http://dx.doi.org/10.1016/j.compstruc.2015.08.013

PUBLISHER

(C) Elsevier

VERSION

AM (Accepted Manuscript)

\section{PUBLISHER STATEMENT}

This work is made available according to the conditions of the Creative Commons Attribution-NonCommercialNoDerivatives 4.0 International (CC BY-NC-ND 4.0) licence. Full details of this licence are available at: https://creativecommons.org/licenses/by-nc-nd/4.0/

\section{LICENCE}

CC BY-NC-ND 4.0

\section{REPOSITORY RECORD}

Dona, Marco, Alessandro Palmeri, and Mariateresa Lombardo. 2015. "Dynamic Analysis of Multi-cracked Euler-bernoulli Beams with Gradient Elasticity”. Loughborough University. https://hdl.handle.net/2134/18910. 


\title{
Dynamic analysis of multi-cracked Euler-Bernoulli beams with gradient elasticity
}

\author{
Marco Donà $^{\mathrm{a}}$, Alessandro Palmeri ${ }^{\mathrm{a}, *}$, Mariateresa Lombardo ${ }^{\mathrm{a}}$ \\ ${ }^{a}$ School of Civil and Building Engineering, Loughborough University, Sir Frank Gibb Building, Loughborough LE11 3TU, England
}

\begin{abstract}
A Galerkin-type approach is presented and numerically validated for the vibration analysis of non-local slender beams with multiple cracks, in which a hybrid gradient elasticity (HGE) model accounts for the microstructural effects. It is shown that: i) a smoother and more realistic profile of beam's rotations is obtained at the damaged locations; ii) independently of support restraints and damage scenarios, only four boundary conditions are required, meaning that the computational effort does not increase with the number of cracks; iii) the microstructural effects become significant when the modal wave lengths are less then about forty times the HGE length-scale parameters.

Keywords: Aifantis' strain gradient, Eringen's stress gradient, Euler-Bernoulli beam, Galerkin method, Hybrid gradient elasticity (HGE), Non-local elasticity
\end{abstract}

\section{Introduction}

Damage often occurs during the service life of structures, and accurate computational models are required to analyse the resulting changes in their performance. Introducing a discrete spring (DS) at the damage position is one of the preferred options for cracked beams and columns, particularly for problems of damage identification, where some iterative processes are required and any little time saving at each iteration corresponds to a significant computational advantage, e.g. when compared to a more detailed 2D/3D finite element (FE) model.

In the traditional application of DSs, a cracked beam or column is ideally split at the damage position, using linear or non-linear springs to transfer the relevant internal forces [1-3], and results into a finite discontinuity in the associated kinematic quantities (rotations and/or axial/transverse displacements). This oversimplifies the smoother behaviour experienced by actual beams at the damaged sections, motivating a recent study by the same authors [4], in which higher-order elasticity theories have been used for the static analysis of non-local slender beams in bending. In this model, a hybrid gradient elasticity (HGE) theory, where both stress gradient and strain gradient enrich the elastic

${ }^{*}$ Corresponding author

Email address: A.Palmeri@Lboro.ac.uk, Dynamics.Structures@Gmail.com (Alessandro Palmeri) 
constitutive law, has been combined with the multi-cracked flexibility model, originally introduced by Palmeri and Cicirello [5] for the classical elasticity. As a result, not only the rotations of the beam's cross section appear smoothed in the neighbourhood of the DS, but size-dependent effects related to the microstructural arrangement of the materials can also be captured, without the higher computational cost of a detailed microstructural model.

Higher-order elasticity theories have been pioneered by Mindlin in the middle of the 20th century [6], but only few decades later they have found two efficient formulations with the work of Eringen [7-9] and Aifantis [10-12]. Applications were initially focused on 2D shell elements or to study wave propagations and crack singularities, and only at the beginning of the 21 st century non-local elasticity theories have found application to $1 \mathrm{D}$ beam elements, mainly because of the interest in the development of CNT (carbon nanotube) systems [13]. Noticeably, similar governing equations can also be used to study composite beams with interlayer slip [14], beams resting on two-parameter elastic foundations [15] and discrete systems [16].

Several papers have been published on the vibration of beams using gradient elasticity theories, for both EulerBernoulli (EB) and Timoshenko beams. Amongst others, Wang and Varadan [17] have developed a gradient-elastic model to study the vibration of both single-walled and double-walled CNTs; Wang et al. [18] have investigated the dynamics of non-local Timoshenko beams, using the Hamilton's principle in conjunction with Eringen's nonlocal (stress-gradient) elasticity theory; Murmu and Adhikari [19, 20] have studied the transverse and longitudinal vibration of nanobeams and nanorods double-systems with distributed longitudinal/transverse springs, employing once again the Eringen's model and highlighting the strong influence of the length-scale parameters on their dynamics. Further work has also been done on different structural configurations, e.g. CNTs with attached buckyballs at tip $[21,22]$, where the additional mass appendix appears to significantly influence both torsional and axial vibrations. The importance of the non-local effects in such circumstances has also been validated with molecular dynamic simulations.

In comparison, limited attention has been paid to the two-parameter HGE model. Zhang et al. [23] have studied the free transverse vibration of slender beams with this constitutive law, providing the analytical solution for simply supported beams, as in this case the sixth-order differential equation of motion can be converted into three second-order differential equations, while for different boundary conditions (BCs) they suggested to resort to numerical methods.

Only a few authors have tackled the problem of gradient-elastic beams with concentrated damage. Loya et al. [24] have used the differential equations governing the dynamics of stress-gradient EB beams for the undamaged segments obtained by splitting the beam at the crack position. The method requires applying four BCs and the four compatibility conditions at the crack location, therefore increasing the size of the problem. Torabi and Dastgerdi [25] have used the same approach for the free vibrations of Timoshenko beams.

Even though effective for stress-gradient elasticity, this solution is not directly applicable to the more general 
HGE theory. Indeed, the problem is ruled in this case by a sixth-order differential equation (not fourth-order, as for the stress-gradient elasticity), therefore additional continuity conditions have to be enforced. The larger size of the problem and the more complicated analytical expressions make this approach unappealing for the HGE theory. The same difficulties hinder the development of a beam-type HGE FE, as two additional degrees of freedom (DoFs) at each node would be required in comparison to the classical elasticity.

In order to overcome these problems, a Galerkin-type approximation is proposed for the transverse vibration analysis of non-local (HGE) multi-cracked slender beams. The assumed shape functions are some closed-form solutions available for conveniently selected static problems, which allow limiting the computational effort, as no additional continuity conditions are required at the crack positions. The proposed approach has been validated against published results for undamaged gradient-elastic beams [23] and multi-cracked beams with classical (local) elasticity [26], and the numerical examples have been used to quantify the effects of the two length-scale parameters (stress-gradient and strain gradient) on the dynamic response of cracked beams.

\section{Multi-damaged Euler-Bernoulli (EB) beam with hybrid gradient elasticity (HGE)}

\subsection{Governing equations}

Let us consider a straight slender beam with bending flexibility $\Gamma(x)=1 / E I(x)$, where the function $E I(x)>0$ is the flexural stiffness and $x$ is the spatial coordinate, ranging from 0 to the length $L$ of the beam.

Resorting to the model recently proposed by the same authors for the static analysis of beams with HGE [4], the constitutive law can be expressed as:

$$
\left\{\begin{array}{l}
\Gamma(x) M(x)=\widetilde{\chi}(x)-\ell_{\varepsilon}^{2} \widetilde{\chi}^{\prime \prime}(x) ; \\
\chi(x)=\widetilde{\chi}(x)-\ell_{\sigma}^{2} \widetilde{\chi}^{\prime \prime}(x),
\end{array}\right.
$$

where the prime ( $($ ) represents the derivative with respect to $x ; M(x)$ is the bending moment (positive if sagging); $\ell_{\varepsilon}>0$ and $\ell_{\sigma}>0$ are the strain-gradient and stress-gradient length-scale parameters, related to the microstructure of the beam; $\widetilde{\chi}(x)$ is the effective non-local curvature, defined as the spatial weight average of the local curvature $\chi(x)$ $[15,23]$. Interestingly, if the two length-scale parameters take the same value, i.e. $\ell_{\sigma}=\ell_{\varepsilon}$, the governing equation for the classical elasticity is recovered, while Eringen's stress-gradient model is obtained for $\ell_{\varepsilon}=0$ and Aifaintis' stress-gradient model for $\ell_{\sigma}=0$.

Using the discrete-spring (DS) model for concentrated damage, the flexibility function $\Gamma(x)$ in Eq. (1) can be written as:

$$
\Gamma(x)=\Gamma_{0}\left[1+F_{\mathrm{b}}(x)\right],
$$


where $\Gamma_{0}$ is the bending flexibility of the undamaged section; while the function $F_{\mathrm{b}}(x)$ includes the effects of the $n$ cracks through Dirac's delta functions $\delta\left(x-\bar{x}_{i}\right)$ centred at each damage position $\bar{x}_{i}$ :

$$
F_{\mathrm{b}}(x)=\sum_{i=1}^{n} \beta_{i} \delta\left(x-\bar{x}_{i}\right)
$$

in which the dimensionless parameter $\beta_{i}$ quantifies the severity of the crack and can be related to the rotational stiffness $K_{i}$ of the DS as [5]:

$$
K_{i}=\frac{E I_{0}}{\beta_{i} L}
$$

Eq. (1) can be solved by imposing two boundary conditions (BCs) on the first derivative of the non-local curvature, which are usually assumed to be zero at the two ends of the beam, i.e. $\widetilde{\chi}^{\prime}(0)=\widetilde{\chi}^{\prime}(L)=0$; and four BCs on the displacement $u$, rotation $\varphi$, bending moment $M$ and shear force $V$, which arise when considering the compatibility equations, $\chi(x)=\varphi^{\prime}(x)=-u^{\prime \prime}(x)$, and the equilibrium equations, $q(x)=-V^{\prime}(x)=-M^{\prime \prime}(x)$, where both $u(x)$ and the distributed load $q(x)$ are positive when downward [4].

\subsection{Proposed approach}

The availability of closed-form exact solutions for the static problem [4] is exploited in this study to develop a novel computational method for the dynamic analysis of EB beams with HGE. Resorting to a Galerkin-type approximation, the main idea here is to use as shape functions the deformed shapes of the beam obtained for a convenient set of point loads, i.e. considering the transverse load as $q(x)=P_{r} \delta\left(x-x_{r}\right)$ for the $r$ th loading case (see Figure 1), which therefore allows the closed-form calculation of the shape functions (see Appendix A). The procedure requires three main steps:

1. Definition of the shape functions (in closed-form);

2. Evaluation of stiffness matrix and mass matrix;

3. Solution of the eigenvalue problem,

which are detailed in the following.

\subsubsection{Definition of the shape functions}

The domain $[0, L]$ is initially split into equal intervals $\Delta x$, defined by the number $N$ of different positions (dummy point-load positions) along the beam. Depending on the BCs:

- $\Delta x=x_{r+1}-x_{r}=\frac{L}{N+1}$, if transverse displacements are prevented at both ends (simply-supported, clampedclamped or propped beams); 


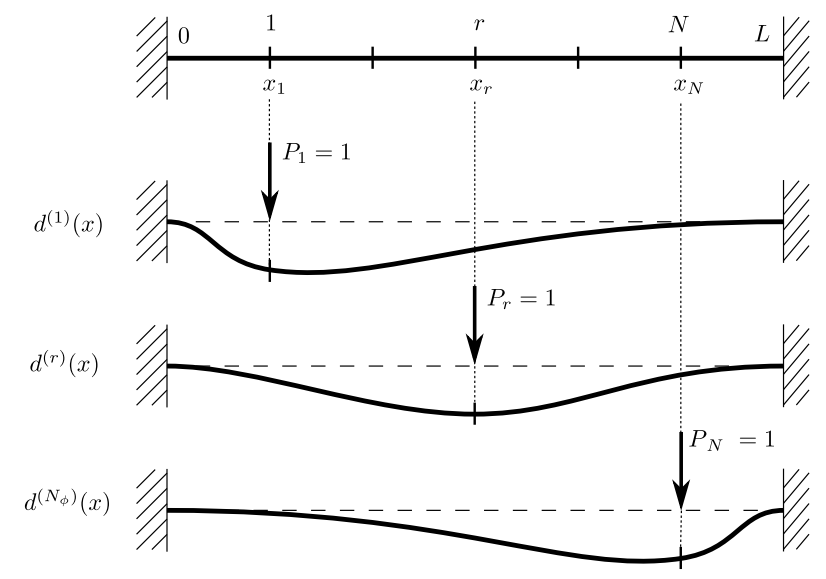

Figure 1: Displacement shape functions for the dynamic analysis

- $\Delta x=x_{r+1}-x_{r}=\frac{L}{N}$, for the cantilever beam.

The approximate expressions for the time-dependent bending moment $M^{*}(x, t)$, curvature $\chi^{*}(x, t)$ and displacement $u^{*}(x, t)$ can then be represented as linear combinations of a set of $N$ shape functions:

$$
\begin{aligned}
& M^{*}(x, t)=\sum_{r=1}^{N} \theta^{(r)}(t) m^{(r)}(x) ; \\
& \chi^{*}(x, t)=\sum_{r=1}^{N} \theta^{(r)}(t) c^{(r)}(x) ; \\
& u^{*}(x, t)=\sum_{r=1}^{N} \theta^{(r)}(t) d^{(r)}(x),
\end{aligned}
$$

where $\theta^{(r)}(t)$ are $N$ time-dependent variables; while $m^{(r)}(x), c^{(r)}(x)$ and $d^{(r)}(x)$ are the assumed shape functions. In s8 detail, the $r$ th transverse displacement function $d^{(r)}(x)$ is the closed-form solution of the beam under a unit point load ${ }_{89} P_{(r)}=1$ applied at the dummy point-load position $x_{r}$ (see Figure 1), and $c^{(r)}(x)$ and $m^{(r)}(x)$ are the corresponding curvature and bending moment, respectively (and $m^{(r)}(x) \neq E I_{0} c^{(r)}(x)$, unless $\ell_{\varepsilon}=\ell_{\sigma}$ ).

It is worth noting here that using these shape functions leads to similar shear force and bending moment diagrams (piecewise constant and piecewise linear, respectively) as in the classical EB beam finite element (FE), where the cubic shape functions for the displacements, differentiated two and three times, correspond to linear $M$ and constant 
For an ideal elastic system without energy dissipation, the $N$ Lagrange's equation of motion can be written as $(r=1, \cdots, N)$ :

$$
\frac{\mathrm{d}}{\mathrm{d} t}\left[\frac{\partial}{\partial \dot{\theta}^{(r)}(t)} \mathcal{L}(t)\right]-\frac{\partial}{\partial \theta^{(r)}(t)} \mathcal{L}(t)=\frac{\partial}{\partial \theta^{(r)}} \mathcal{W}(t),
$$

where $\theta^{(r)}(t)$ and $\dot{\theta}^{(r)}(t)$ are the generalised displacements and velocities, respectively; while $\mathcal{L}(t)$ is the so-called Lagrangian function, defined as the difference between the kinetic energy $\mathcal{T}(t)$ and the potential energy $\mathcal{V}(t)$ :

$$
\mathcal{L}(t)=\mathcal{T}(t)-\mathcal{V}(t)
$$

which can be evaluated as:

$$
\begin{gathered}
\mathcal{V}(t)=\frac{1}{2} \int_{0}^{L} M(x, t) \chi(x, t) \mathrm{d} x \\
\mathcal{T}(t)=\frac{1}{2} \rho A \int_{0}^{L} \dot{u}^{2}(x, t) \mathrm{d} x,
\end{gathered}
$$

where $\rho$ is the mass density of the material and $A$ is the cross sectional area; while the external work is:

$$
\mathcal{W}(t)=\frac{1}{2} \int_{0}^{L} q(x, t) u(x, t) \mathrm{d} x
$$

By using the approximate expressions of the field variables of Eqs. (5), the different forms of energy can be rewritten as:

$$
\begin{gathered}
\mathcal{V}(t)=\frac{1}{2} \sum_{r=1}^{N} \sum_{s=1}^{N} \theta^{(r)}(t) \theta^{(s)}(t) \int_{0}^{L} m^{(r)}(x) c^{(s)}(x) \mathrm{d} x \\
\mathcal{T}(t)=\frac{1}{2} \rho A \sum_{r=1}^{N} \sum_{s=1}^{N} \dot{\theta}^{(r)}(t) \dot{\theta}^{(s)}(t) \int_{0}^{L} d^{(r)}(x) d^{(s)}(x) \mathrm{d} x \\
\mathcal{W}(t)=\frac{1}{2} \sum_{r=1}^{N} \theta^{(r)}(t) \int_{0}^{L} q(x) d^{(r)}(x) \mathrm{d} x .
\end{gathered}
$$

After some algebra, substitution of Eqs. (10) into Eq. (6) yields to a system of ordinary differential equations, which can be posed in a compact matrix form as:

$$
\mathbf{M} \cdot \ddot{\boldsymbol{\theta}}(t)+\mathbf{K} \cdot \boldsymbol{\theta}(t)=\mathbf{F}(t),
$$

where $\boldsymbol{\theta}(t)=\left\{\theta^{(1)}(t), \cdots, \theta^{(N)}(t)\right\}^{\top}$ is the array of the Lagrange variables (the superscripted symbol $\top$ being the transpose operator); $\mathbf{F}(t)=\left\{F^{(1)}(t), \cdots, F^{(N)}(t)\right\}^{\top}$ is the array of the equivalent external loads, where the $r$ th element 
is given by:

$$
F_{(r)}(t)=\int_{0}^{L} q(x, t) d^{(r)}(x) \mathrm{d} x
$$

while $\mathbf{K}$ and $\mathbf{M}$ are the stiffness and mass matrices respectively, whose elements ( $r$ th row and $s$ th column) are so defined:

$$
\begin{gathered}
K_{r s}=\int_{0}^{L} m^{(r)}(x) c^{(s)}(x) \mathrm{d} x ; \\
M_{r s}=\rho A \int_{0}^{L} d^{(r)}(x) d^{(s)}(x) \mathrm{d} x .
\end{gathered}
$$

\section{2.3. Eigenvalue problem}

In order to investigate the free vibration of multi-cracked HGE beams, Eq. (11) is studied without any external force, i.e. with $\mathbf{F}(t)=\mathbf{0}$ :

$$
\mathbf{M} \cdot \ddot{\boldsymbol{\theta}}(t)+\mathbf{K} \cdot \boldsymbol{\theta}(t)=\mathbf{0},
$$

whose $i$ th harmonic solution $\boldsymbol{\theta}_{i}(t)$ depends on the natural circular frequency $\omega_{i}$ and the associated array $\mathbf{a}_{i}=\left\{a_{i}^{(1)}, \cdots, a_{i}^{(N)}\right\}^{\top}$, containing the generalised coordinates of the $i$ th modal shape of the beam $(i=1, \cdots, N)$ :

$$
\boldsymbol{\theta}_{i}(t)=\mathbf{a}_{i} \sin \left(\omega_{i} t\right)
$$

Substituting Eq. (16) into Eq. (15) leads to the generalised eigenproblem:

$$
\mathbf{K} \cdot \mathbf{a}_{i}=\omega_{i}^{2} \mathbf{M} \cdot \mathbf{a}_{i} .
$$

Once the eigenvector $\mathbf{a}_{i}$ is known, the $i$ th modal shape can be expressed as:

$$
\phi_{i}(x)=\mathbf{a}_{i}^{\top} \mathbf{d}(x),
$$

where $\mathbf{d}(x)=\left\{d^{(1)}(x), \cdots, d^{(N)}(x)\right\}^{\top}$ is the $N$-dimensional array of the shape functions introduced to approximate the solution. Table 1 summarises the key steps needed for the implementation of the proposed approach.

\section{Numerical examples}

The dynamic analysis of three cracked non-local beams with different BCs has been carried out with the computational software Mathematica [27]. To the authors' best knowledge, the exact analytical solution is not yet available 
Table 1: Solution procedure for the dynamic problem

1. Collect all the data for the beam (namely, $E, I_{0}, L, n, \bar{x}_{i}, \beta_{i}$, classical BCs) including the microstructural parameters $\left(\ell_{\varepsilon}, \ell_{\sigma}\right)$

2. Choose the $N$ positions of the point loads ( $N=$ number of shape functions)

3. For each position $x_{r}$ of the point load, evaluate:

(a) displacement function $d^{(r)}(x)$

(b) curvature function $c^{(r)}(x)$

(c) bending moment function $m^{(r)}(x)$

4. Evaluate:

(a) each term $K_{r s}$ of the stiffness matrix (see Eq. (13))

(b) each term $M_{r s}$ of the mass matrix (see Eq. (14))

5. Solve the eigenproblem $\left[\mathbf{K}-\omega_{i}^{2} \mathbf{M}\right] \cdot \mathbf{a}_{i}=\mathbf{0}$

in the literature, and so the two cases of undamaged gradient-elastic beams [23] and of multi-damaged local-elastic beams [26] have been used when possible for validation purposes.

As discussed in Ref. [13], length-scale parameters can be related to constitutive or geometrical properties of the material $[28,29]$ or quantified using experimental techniques [30]. In line with the idea that length-scale parameters capture key features of the material at sub-scale, the effects of $\ell_{\varepsilon}$ and $\ell_{\sigma}$ on the dynamic response of the damaged beams have been also quantified. The case of $\ell_{\varepsilon}=\ell_{\sigma}$ has been considered to retrieve the classical elasticity solution. For completeness, the analysis has been extended to the range $\ell_{\varepsilon}<\ell_{\sigma}$, which has been defined as physically inconsistent due to the counter-load deformations observed in the static analysis of the beams [4]. In any case, since these two parameters are linked to the beam's microstructure, their values cannot exceed the beam's length, i.e. $0 \leq \ell_{\varepsilon}<L$ and $0 \leq \ell_{\sigma}<L$. The convergence for an increasing number of shape functions has also been investigated.

For the sake of generality, the results are presented as much as possible in terms of dimensionless quantities, e.g. the ratio $\omega_{i} / \omega_{i, \text { loc }}$ between the $i$ th frequency of the non-local beam and the $i$ th frequency of the classical (local) one.

\subsection{First example - Cantilever beam with a single damage}

A cantilever beam of length $L$ is studied in the first example (see Figure 2). The beam is clamped at $x=0$ and has a single crack at the position $x_{1}=L / 3$, in which the dimensionless parameters of the rotational spring is $\beta_{1}=0.1$ (see Eqs. (3) and (4)).

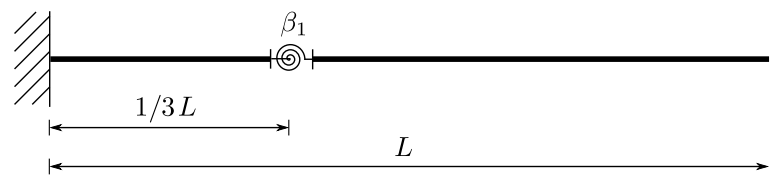

Figure 2: Example one - Cantilever beam with a concentrated single damage 


\begin{tabular}{c|c|c|c}
\hline$\omega_{1, \mathrm{loc}}$ & $\omega_{2, \mathrm{loc}}$ & $\omega_{3, \mathrm{loc}}$ & $\omega_{4, \mathrm{loc}}$ \\
\hline 0.528 & 3.398 & 9.2015 & 19.214 \\
\hline
\end{tabular}

Table 2: Example one - First four natural frequencies for the local elasticity model

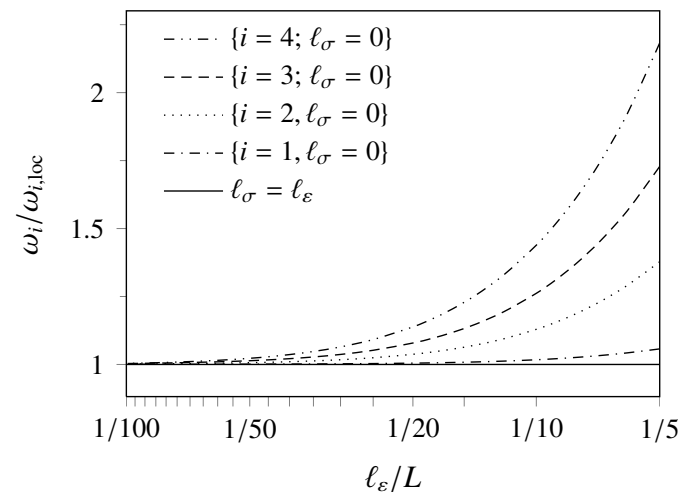

(a)

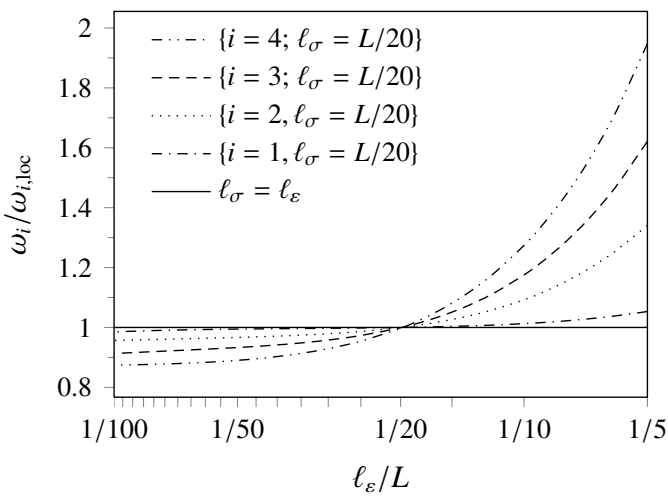

(b)

Figure 3: Example one - Normalised natural frequencies $\omega_{i} / \omega_{i, \text { loc }}$ of the cracked cantilever beam for $\ell_{\sigma}=0$ (a) and $\ell_{\sigma}=L / 20$ (b), computed with $N=16$

Following the procedure summarised in Table 1, a set of $N$ different concentrated loads $P_{r}=1$ pointing downward is applied along the beam, each one giving a different shape function for the displacement $d^{(r)}(x)$, the local curvature $c^{(r)}(x)=-d^{\prime \prime(r)}(x)$ and the bending moment $m^{(r)}(x)$. The integration constants $C_{0}^{(r)}, C_{1}^{(r)}, C_{2}^{(r)}$ and $C_{3}^{(r)}$ are evaluated by solving the four equations that result from applying the BCs for the internal forces at the free end $\left(m^{(r)}(L)=\right.$ $\left.m^{\prime(r)}(L)=0\right)$ and the kinematic quantities at the fixed end $\left(d^{(r)}(0)=d^{\prime(r)}(0)=0\right)$; the other two constants $C_{4}^{(r)}$ and $C_{5}^{(r)}$ are evaluated by assuming the stationarity of the effective non-local curvature at both ends of the beam $\left(\tilde{c}^{\prime(r)}(0)=\tilde{c}^{\prime(r)}(L)=0\right)$.

\subsubsection{Effects of the length-scale parameters}

Modal frequencies $\omega_{i}$ and modal shapes $\phi_{i}(x)$ obtained with the proposed formulation for different combinations of the length-scale parameters $\ell_{\varepsilon}$ and $\ell_{\sigma}$ have been compared with those of the classical elasticity theory. The lin-log plots of Figure 3(a) show how the first four normalised modal frequencies $\omega_{i} / \omega_{i, \text { loc }}$ vary with the normalised straingradient parameter $\ell_{\varepsilon} / L$ (between $1 / 100$ and $1 / 5$ ), while the stress-gradient parameter is $\ell_{\sigma}=0$ (Aifantis' model). Table 2 collects the reference values of the modal frequencies $\omega_{i, \text { loc }}$, which in all the numerical examples have been computed using the exact closed-form expressions derived by Caddemi and Caliò [26].

Independently of the value of the strain-gradient parameter, the higher the modal frequencies, the larger the relative effect of the gradient elasticity; in particular, for the fourth frequency $\omega_{4}$, the increment with respect to $\omega_{4, \text { loc }}$ is larger 


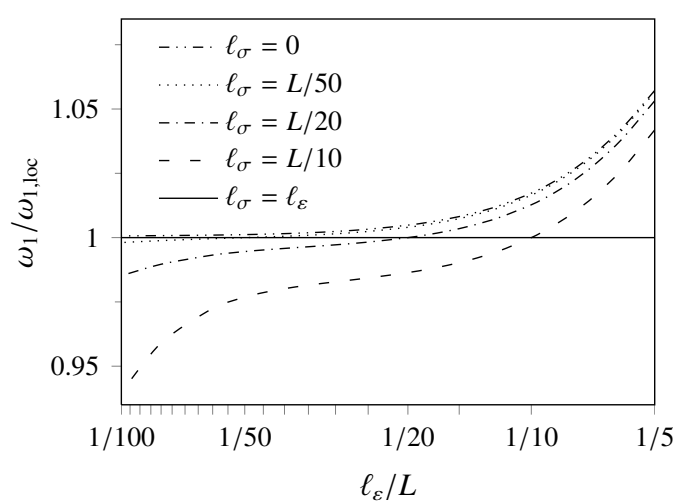

(a)

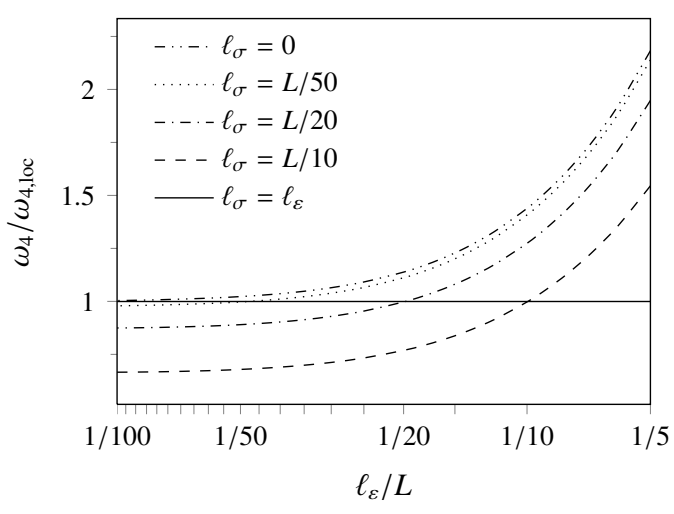

(b)

Figure 4: Example one - Ratio $\omega_{i} / \omega_{i, \text { loc }}$ for the first $(i=1)$ (a) and fourth $(i=4)$ (b) natural frequencies for four different values of the stressgradient parameter $\ell_{\sigma}$, computed with $N=16$

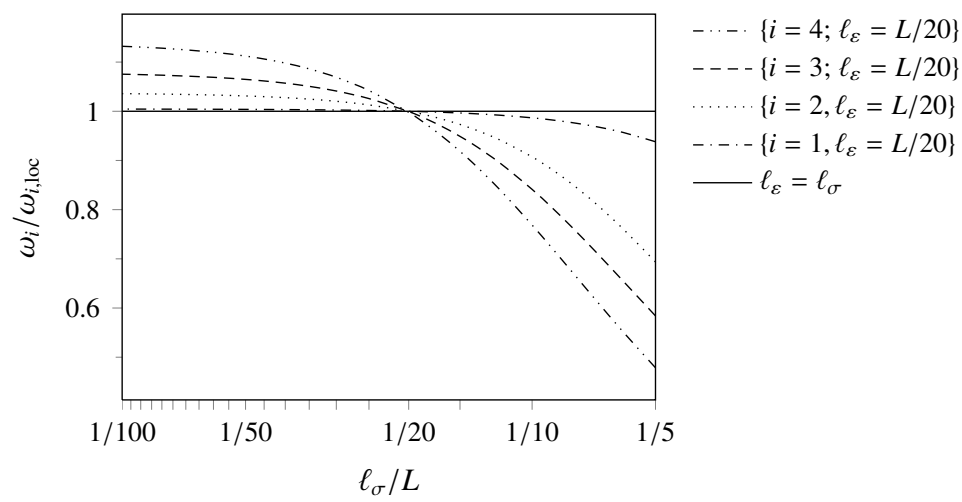

Figure 5: Example one - First four normalised frequencies $\omega_{i} / \omega_{i, \text { loc }}$ for $\ell_{\varepsilon}=L / 20$, computed with $N=16$ $\ell_{\varepsilon}=\ell_{\sigma}$, and increasing $\ell_{\sigma}$ reduces the frequencies.

Interestingly, one can observe that for $\ell_{\varepsilon}<\ell_{\sigma}$ the modal frequencies reduce, i.e. $\omega_{i}<\omega_{i \text {,loc }}$, meaning that the HGE beam is less stiff than the local counterpart; the opposite happens for $\ell_{\varepsilon}>\ell_{\sigma}$, as in this case the modal frequencies increase, i.e. $\omega_{i}>\omega_{i, \text { loc }}$. More generally, for a given value of the stress-gradient length-scale parameter $\ell_{\sigma}$, the larger $\left|\ell_{\varepsilon}-\ell_{\sigma}\right|$, the more significant tends to be the variation in the modal frequencies. 
Additionally, for a given set of length-scale parameters $\left\{\ell_{\varepsilon}, \ell_{\sigma}\right\}$, the higher the mode of vibration, the larger tends to be the variation in the modal frequency. For the cantilever beam under consideration and the range of selected parameters, there is a maximum reduction in first modal frequency $\omega_{1}$ of about $6 \%$ for $\ell_{\varepsilon}=L / 100$ and $\ell_{\sigma}=L / 10$, while the maximum increase of about $5 \%$ is seen for $\ell_{\varepsilon}=L / 5$ and $\ell_{\sigma}=0$ (see Figure 4(a)). Larger variation are observed for the fourth modal frequency (see Figure 4(b)), in which for the same values of length-scale parameters $\omega_{4}$ reduces to about $30 \%$ less than $\omega_{4, \text { loc }}$ (for $\ell_{\varepsilon}=L / 100$ and $\ell_{\sigma}=L / 10$ ) and increases to about $120 \%$ more than $\omega_{4, \text { loc }}$ (for $\ell_{\varepsilon}=L / 5$ and $\ell_{\sigma}=0$ ).

Figure 5 shows the variation of the first four natural frequencies when the strain-gradient parameter takes the constant value $\ell_{\varepsilon}=L / 20$ and $\ell_{\sigma}$ varies between $L / 100$ and $L / 5$. As usual, the intersection point represents the solution for $\ell_{\varepsilon}=\ell_{\sigma}=L / 20$ and is equivalent to the classical elasticity solution. For both left and right side of the plot (corresponding to $\ell_{\sigma}<\ell_{\varepsilon}$ and $\ell_{\sigma}>\ell_{\varepsilon}$, respectively), the higher the frequency, the larger are the non-local effects. For instance, for $\ell_{\varepsilon}=L / 20$ and $\ell_{\sigma}=L / 5$, the fourth modal frequency of the non-local beam is about $50 \%$ lower than the corresponding local one.

The effects of the damage parameter $\beta_{1}$ on the first four natural frequencies are illustrated within Figure 6, assuming $\ell_{\sigma}=L / 20$ and five different values of the strain-gradient parameter, $\ell_{\varepsilon}=\{L / 40, L / 30, L / 20, L / 10, L / 5\}$. When compared with the local-elastic case $\left(\ell_{\sigma}=\ell_{\varepsilon}=L / 20\right)$, the numerical results show the material to soften for $\ell_{\varepsilon}<\ell_{\sigma}$, leading to lower frequencies; on the contrary, the material becomes stiffer for $\ell_{\varepsilon}>\ell_{\sigma}$. Furthermore, independently of the microstructural parameters $\ell_{\varepsilon}$ and $\ell_{\sigma}$, a significant reduction of the frequencies $\omega_{1}, \omega_{2}$ and $\omega_{3}$ is observed when $\beta_{1}$ increases. A less pronounced reduction is seen in the fourth modal frequency $\omega_{4}$ (Figure $6(\mathrm{~d})$ ), as the associated modal shape has a small value of the bending moment at the cracked cross section $(x=L / 3)$, and for this reason the concentrated damage has very little effect on the fourth mode.

\subsubsection{Modal shapes}

The above analysis of the modal frequencies proceeds with studying the effects of the microstructural parameters $\ell_{\varepsilon}$ and $\ell_{\sigma}$ on the modal shapes of the cracked cantilever beam. Figure 7 shows the first four modal shapes for four different combinations of the length-scale parameters $\ell_{\sigma}=L / 20$ and $\ell_{\varepsilon}=\{L / 40, L / 20, L / 10, L / 5\}$. As usual, the solid line represents the local case, retrieved for $\ell_{\varepsilon}=\ell_{\sigma}=L / 20$. The first observation is that the first modal shape $\phi_{1}(x)$ is scarcely affected by the microstructural parameters, while their effects become increasingly more significant in the higher modes of vibration. Interestingly, Figures 7(b), (c) and (d) reveal that the $i$ th modal shape (with $i \geq 2$ ) has $i-1$ nodes, i.e. points (other than the fixed end at $x=0$ ) where the family of curves $\phi_{i}(x)$ tends to pass, independently of the strain gradient parameter $\ell_{\varepsilon}$. 

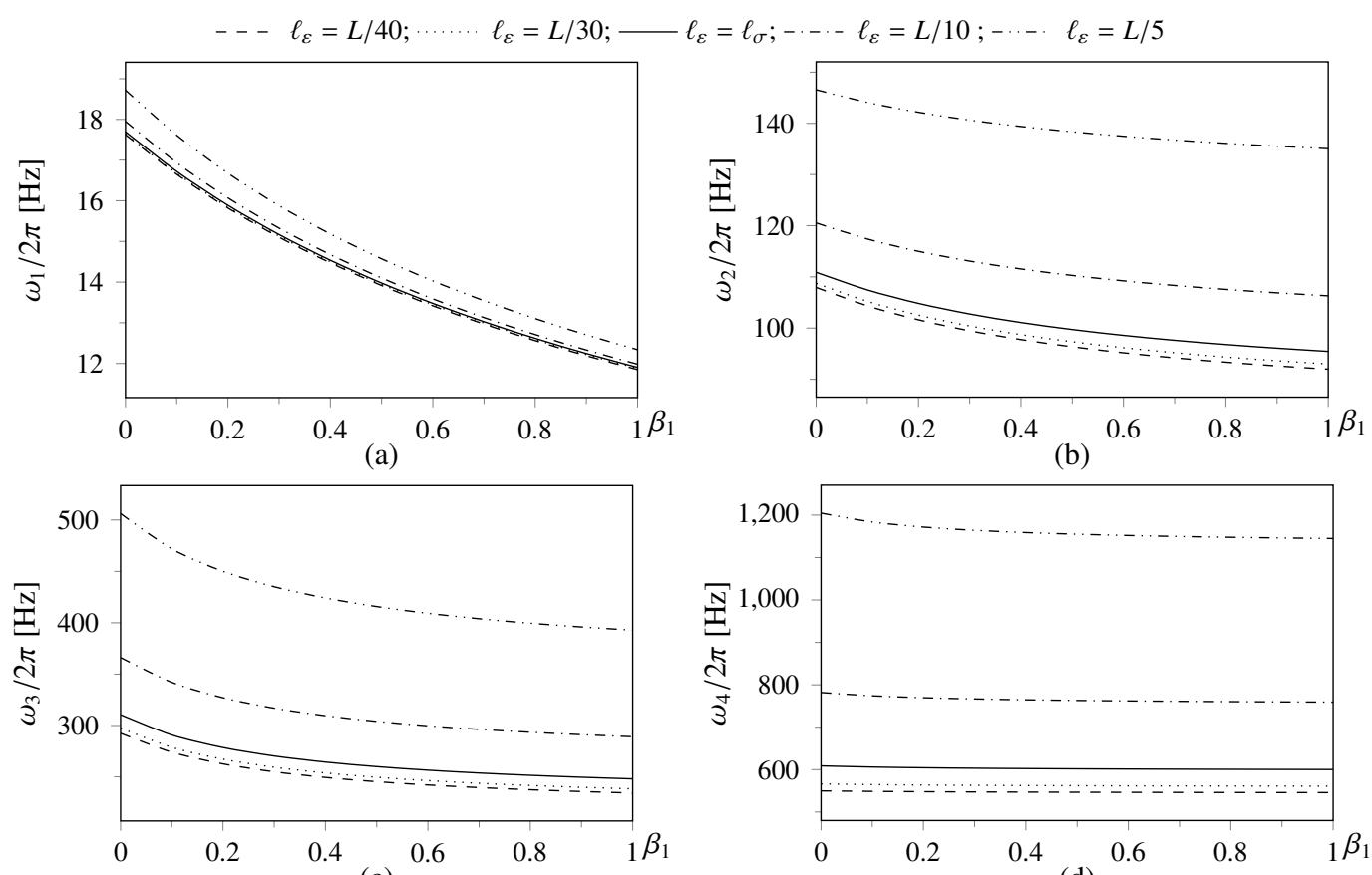

(c)

(d)

Figure 6: Example one - First four modal frequencies $\omega_{i}$ against the damage parameter $\beta_{1}$ for $\ell_{\sigma}=L / 20$ and four different values of the straingradient parameter $\ell_{\varepsilon}=\{L / 40 ; L / 30 ; L / 20 ; L / 10 ; L / 5\}$, computed with $N=16$
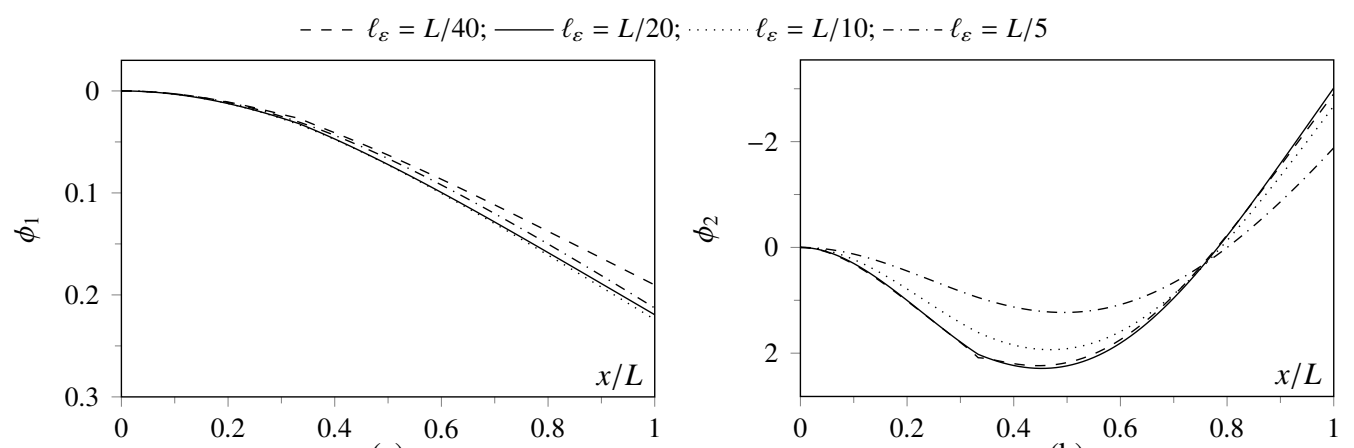

(a)
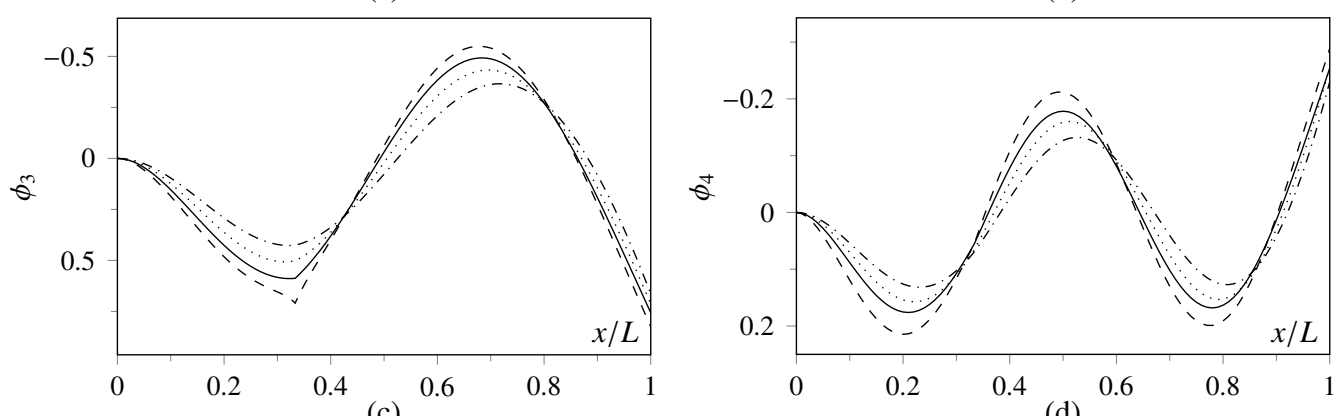

Figure 7: Example one - First four modal shapes for $\ell_{\sigma}=L / 20$ and four different values of the strain-gradient parameter $\ell_{\varepsilon}=\{L / 40 ; L / 20 ; L / 10$; $L / 5\}$, computed with $N=8$ 


\subsubsection{Convergence}

In order to check the efficiency of the proposed method, the convergence of the frequency error against the number of shape functions has been investigated. Since the exact dynamic solution of the cracked hybrid non-local beam model is not available, the frequency obtained using a higher number of shape functions $(N=64)$ has been assumed as a reference value for this study. The dimensionless measure of frequency error for the $i$ th mode has been defined as:

$$
\varepsilon_{i}(N)=\frac{\left|\omega_{i}(N)-\omega_{i, \text { ref }}\right|}{\omega_{i, \text { ref }}},
$$

where $\omega_{i \text {,ref }}=\omega_{i}(N=64)$, and the trend of $\varepsilon_{i}(N)$ is shown for the first four modal frequency as part of Figure 8 .

First of all, one can observe that the relative error assumes values lower than $2 \%$ for all the cases investigated, where the number of elements range from 6 to 64 elements, and this can then be considered as highly acceptable in most engineering simulations. Furthermore, all the graphs show the convergence to the reference solution for an increasing number of shape functions. In particular, the relative error of the first three modal frequencies is already lower than $1 \%$ when six shape functions are adopted, whereas for the fourth frequency it reduces from $2 \%$ to $1 \%$ with $N=16$. Interestingly, the error does not decreases monotonically for the third and fourth modal frequency, due to the fact that increasing the number of dummy point loads $N$ generally results in different shape functions being used, rather than to an enlargement of the set of shape functions, unless the interval $\Delta x$ is consecutively split into an integer number of smaller intervals (e.g. $\Delta x / 2, \Delta x / 4$, etcetera). However, for this example it is clear that using 16 points (i.e. $\Delta x=L / 16$ ) ensures that accurate results are obtained for the first four natural frequencies.

\subsection{Second example - Simply-supported beam with two cracks}

For the second numerical example, a simply supported beam of length $L$ pinned at $x=0$ and $x=L$ has been considered (see Figure 9). The beam has two cracks at $x_{1} / L=3 / 10$ and $x_{2} / L=4 / 7$, modelled with two rotational springs with the same dimensionless damage parameter $\beta_{1}=\beta_{2}=0.1$.

\begin{tabular}{c|c|c|c}
\hline$\omega_{1, \text { loc }}$ & $\omega_{2, \text { loc }}$ & $\omega_{3, \text { loc }}$ & $\omega_{4, \text { loc }}$ \\
\hline 1.366 & 5.685 & 13.327 & 23.451 \\
\hline
\end{tabular}

Table 3: Example two - First four natural frequencies for the local elasticity model $\omega_{i, \text { loc }}$

\subsubsection{Effects of the length-scale parameters}

As in the previous example, the first four modal frequencies of the HGE beam have been computed and compared with those of the classical elasticity, whose values are given in Table 3. 


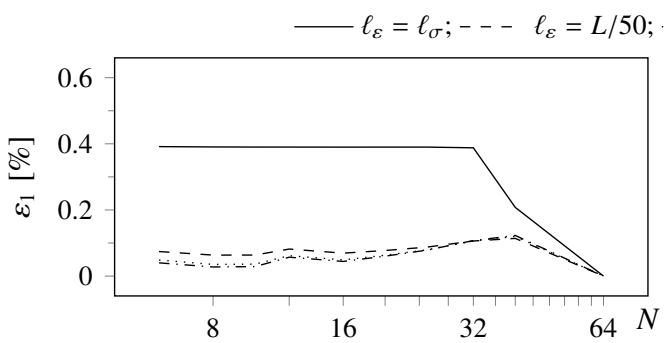

(a)

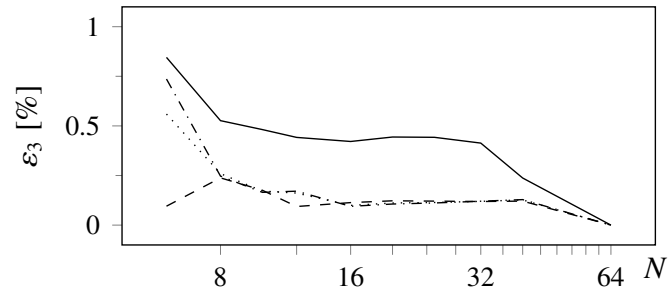

(c)

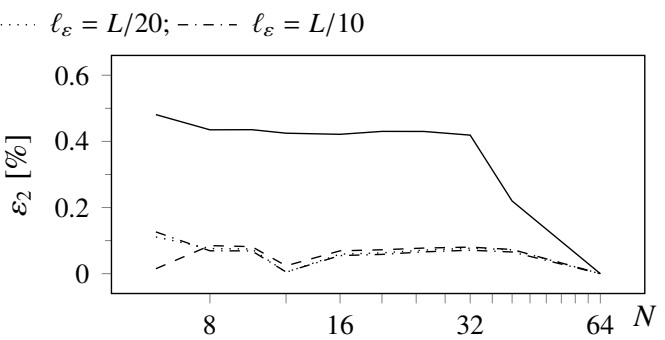

(b)

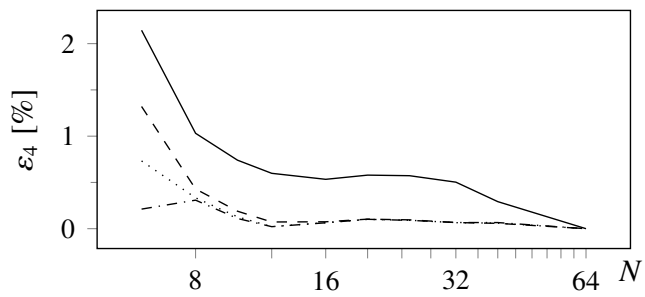

(d)

Figure 8: Example one - Convergence diagram of the normalised frequency error $\varepsilon_{i}$ for $\ell_{\sigma}=0$ and four different values of the strain length-scale parameter $\ell_{\varepsilon}=\{0 ; L / 50 ; L / 20 ; L / 10\}$

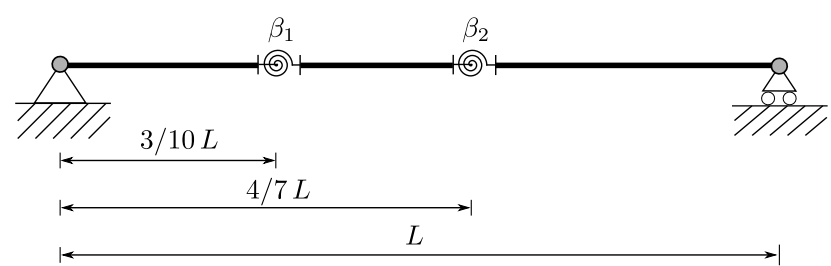

Figure 9: Example two - Simply supported beam with two cracks

The effects of $\ell_{\varepsilon}$ on the Aifantis' model (with $\ell_{\sigma}=0$ ) is shown within Figure 10(a), revealing that, also in this case, the higher the mode, the larger the effects on the modal frequencies, e.g. more than $100 \%$ of increase for the fourth modal frequency when the ratio $\ell_{\varepsilon}$ passes from $L / 100$ to $L / 5$. Figure 10(b) displays the results of the same analysis with the HGE model, with stress-gradient parameter taken as $\ell_{\sigma}=L / 20$. Once again, higher frequencies are more affected by the variation in strain length-scale; furthermore for $\ell_{\varepsilon}=\ell_{\sigma}=L / 20$ the frequencies are equal to those of the local beam; for $\ell_{\varepsilon}<\ell_{\sigma}$ the frequencies of the non-local beam are lower; whereas the opposite happens for $\ell_{\varepsilon}>\ell_{\sigma}$.

Aimed at quantifying when the non-local effects become dynamically significant, i.e. when they can be important from an engineering point of view, the magnified plot of Figure 11 shows that for $\ell_{\varepsilon}>L / 20$ the first modal frequency of the Aifantis' model $\left(\ell_{\sigma}=0\right)$ is more than $1 \%$ higher than the value obtained with the classical elasticity theory; interestingly, a smaller microstructural parameter, i.e. $\ell_{\varepsilon} \simeq L / 45$, is enough to increase by $1 \%$ the second modal frequency; even smaller length-scale parameters, namely $\ell_{\varepsilon} \simeq L / 65$ and $\ell_{\varepsilon} \simeq L / 85$, produce an increase of about $1 \%$ in the third and fourth modal frequencies. Moving from these observations, Table 4 compares the ratio between 


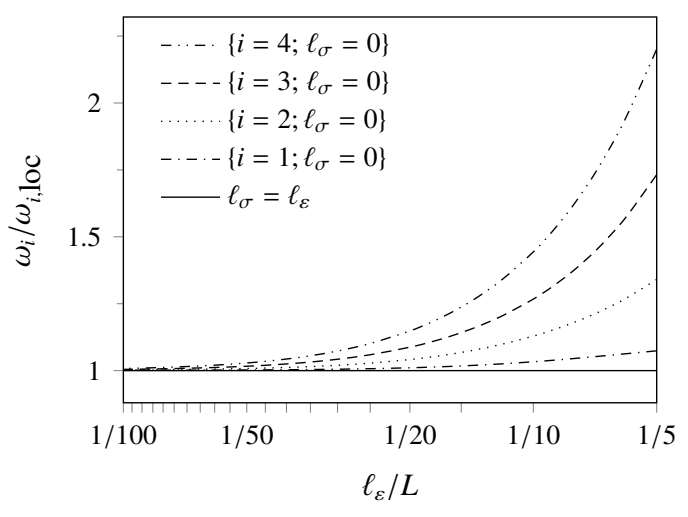

(a)

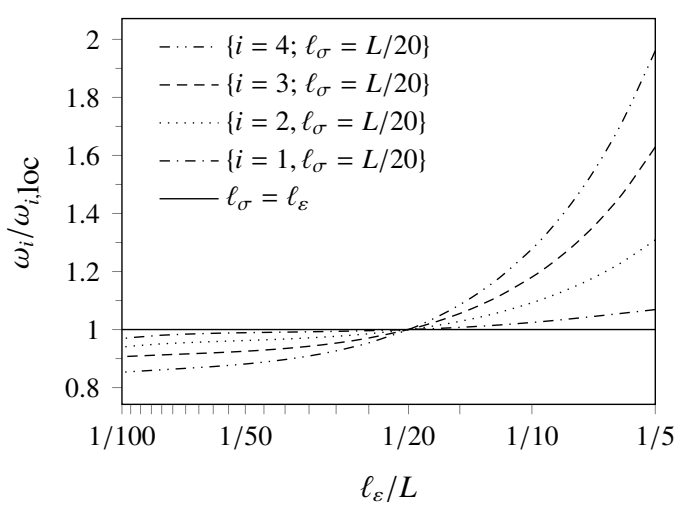

(b)

Figure 10: Example two - Normalised natural frequencies $\omega_{i} / \omega_{i, \text { loc }}$ for $\ell_{\sigma}=0$ (a) and $\ell_{\sigma}=L / 20$ (b), computed with $N=15$

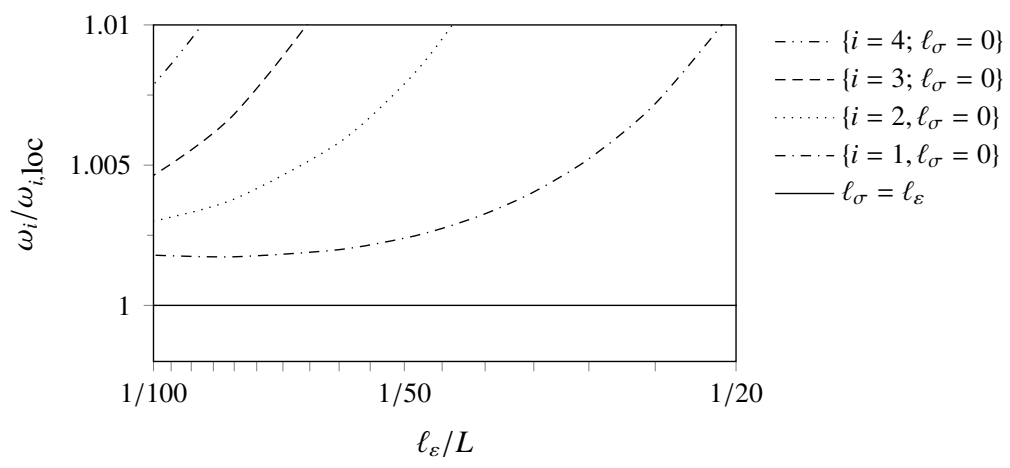

Figure 11: Example two - Magnified view of Figure 10(a) for $L / 100 \leq \ell_{\varepsilon} \leq L / 20$ and $\omega_{i} \leq 1.01 \omega_{i, \text { loc }}$

the average wave length in the first four modes of vibration, $\lambda_{i}=L / i(i=1,2,3,4)$, against the value of the strain length-scale parameter $\ell_{\varepsilon}^{*}$ for which an effect of about $1 \%$ is seen when comparing the Aifantis' model $\left(\ell_{\sigma}=0\right)$ with the classical elasticity $\left(\ell_{\varepsilon}=\ell_{\sigma}\right)$. Interestingly the table reveals that, independently of the particular mode being considered, the microstructural parameters start to be noticeable (with effects higher than $1 \%$ ) when the ratio $\lambda_{i} / \ell_{\varepsilon}^{*}$ is less than about 40. This explains why higher modes of vibration are more affected by the gradient-elastic constitutive law, as they tend to have wave lengths more comparable with the microstructural length-scales.

\subsubsection{Modal shapes}

The dynamic analysis of the cracked beam of Figure 9 proceeds with studying the modal shapes, looking at the effects of different microstructural parameters. Figures 12 to 15 display the first four modal shapes in terms of transverse displacement (a), rotation $\varphi_{i}(\mathrm{~b})$, bending moment $M_{i}(\mathrm{c})$ and shear force $V_{i}(\mathrm{~d})$. All the results are obtained in this case using $N=7$ dummy load points (i.e. $\Delta x=L / 8$ ). In particular, the rotations' profiles for the different microstructural parameters highlight the smoothing effect due to the inclusion of the HGE theory when $\ell_{\varepsilon}>\ell_{\sigma}$, while 


\begin{tabular}{c|ccc}
\hline & $\lambda_{i}$ & $\ell_{\varepsilon}^{*}$ & $\lambda_{i} / \ell_{\varepsilon}^{*}$ \\
\hline $1^{\text {st }}$ mode & $2 L$ & $L / 20$ & 40 \\
$2^{\text {nd }}$ mode & $L$ & $L / 45$ & 45 \\
$3^{\text {rd }}$ mode & $(2 / 3) L$ & $L / 65$ & 43.3 \\
$4^{\text {th }}$ mode & $L / 2$ & $L / 85$ & 42 \\
\hline
\end{tabular}

Table 4: Example two - Modal wave lengths $\lambda_{i}$ and strain-gradient parameters $\ell_{\varepsilon}^{*}$ showing a $1 \%$ increase in the first four modal frequencies with the Aifaintis's model $\left(\ell_{\sigma}=0\right)$

the opposite happens when $\ell_{\varepsilon}<\ell_{\sigma}$, as the finite jump of rotations at the cracked sections is amplified in comparison with the classical elasticity, leading to angular points in the deformation profiles.

Figures 12 to 15 allow also showing that, as a direct consequence of the proposed computational approach, the shear force diagrams of the modal shapes are piecewise constant functions, while the bending moment are piecewise linear functions. This is consistent with the application of dummy point loads at $x_{r}=r \Delta x(r=1,2, \cdots, N)$, which induces finite jumps in the shear force diagram and sudden changes in the slope of the bending moment diagram. It is worth stressing here that these diagrams are qualitatively similar to those that are typically obtained when the FE method is used for the dynamic analysis of slender beams with classical (local) elasticity, where cubic shapes in terms of displacements lead to quadratic rotations, linear curvatures and bending moments and constant shear forces within each beam element. The proposed method has then the advantage to ensure the same level of approximation as the classical FE method does for classical (local) beams, with inevitably a better level of approximation in terms of displacements rather than internal forces. Furthermore, a recommendation can be made to take $\Delta x \leq \lambda_{i} / 8$ to achieve a satisfactory representation of bending and shear stresses in the beam.

\subsubsection{Validation of the model}

In order to validate the proposed model, the theoretical solution for the undamaged HGE beam as presented by Zhang et al. [23], has been compared with the results of the proposed Galerkin-type approach. As already noted in Ref. [4], the behaviour of the hybrid non-local model can be considered physically inconsistent for $\ell_{\varepsilon}<\ell_{\sigma}$ because of cusps centred at the crack position, as can be observed in the rotation diagrams shown in the top-right graphs within Figures 12 to 15 . In particular, Table 5 reports the first four frequencies of a simply supported undamaged HGE with two different combinations of length-scale parameters, namely $\left\{\ell_{\varepsilon}=1 / 20, \ell_{\sigma}=L / 5\right\}$ and $\left\{\ell_{\varepsilon}=1 / 20, \ell_{\sigma}=L / 10\right\}$, as computed with the analytical expression by Zhang et al. [23] and with the proposed procedure with $N=31$ shape functions. The comparison shows that the two methods are in excellent agreement, the maximum inaccuracy being less than $0.3 \%$, which therefore contributes to further validate the proposed approach. 

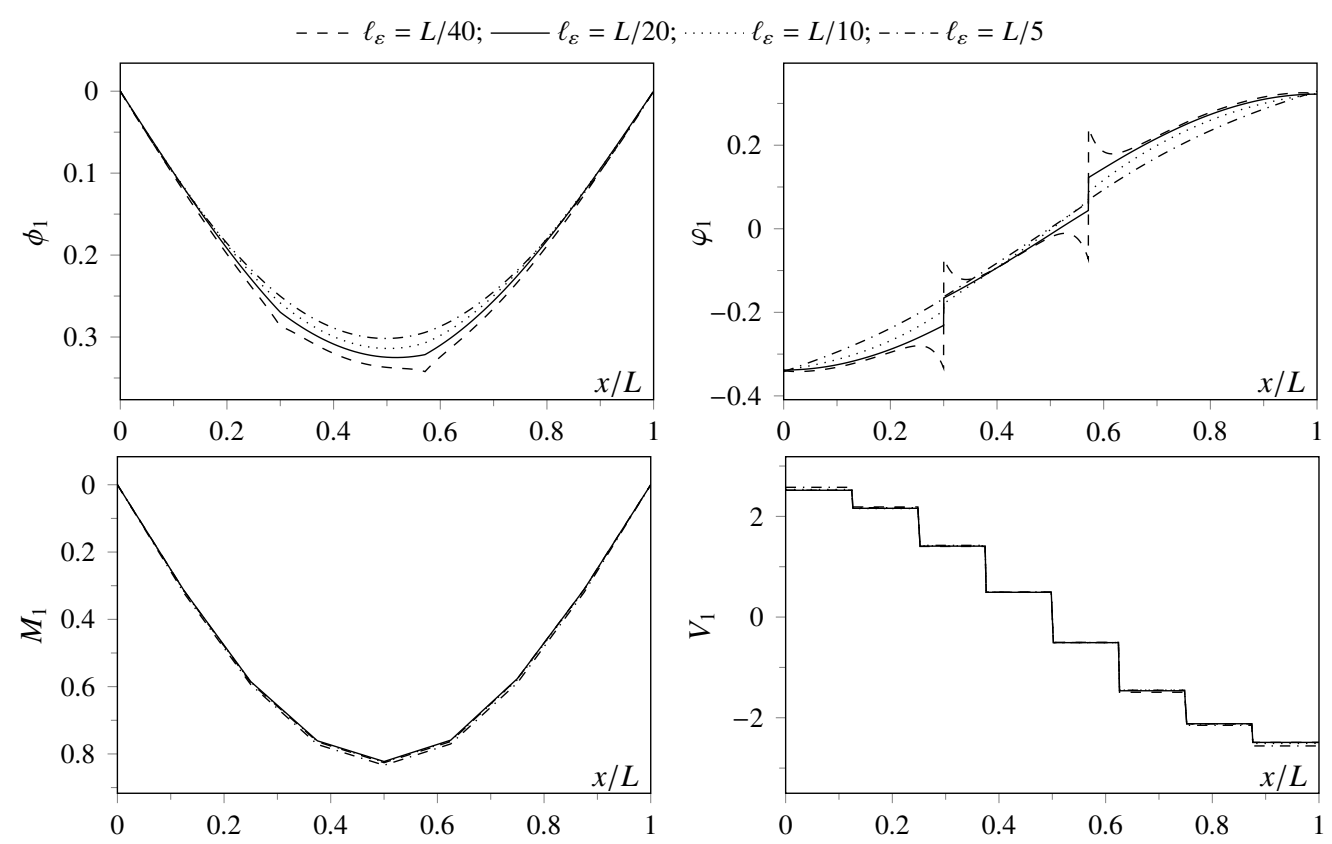

Figure 12: Example two - Deflections, rotations, shear force and bending moment diagrams of the first mode for $\ell_{\sigma}=L / 20$ and four different values of the strain length-scale parameter $\ell_{\varepsilon}=\{L / 40 ; L / 20 ; L / 10 ; L / 5\}$, computed with $N=7$
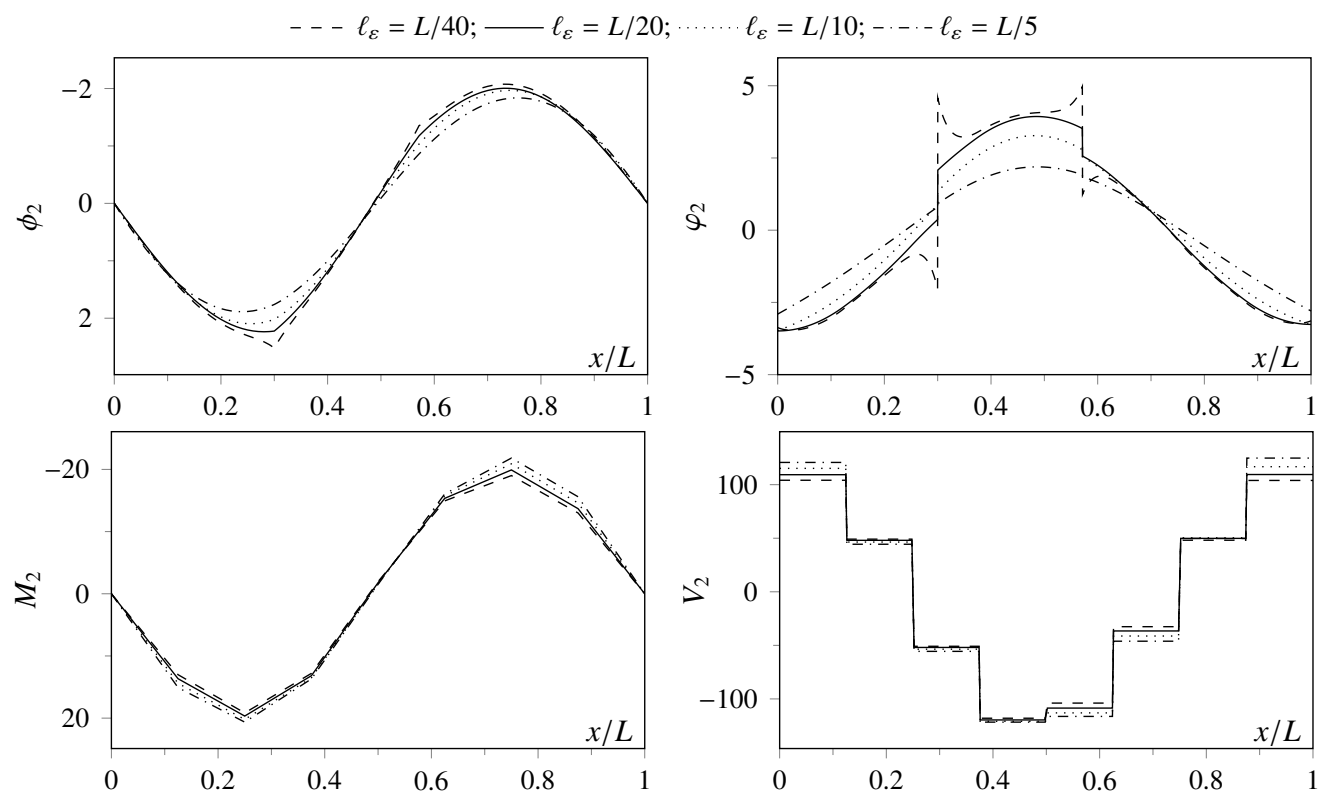

Figure 13: Example two - Deflections, rotations, shear force and bending moment diagrams of the second mode for $\ell_{\sigma}=L / 20$ and four different values of the strain length-scale parameter $\ell_{\varepsilon}=\{L / 40 ; L / 20 ; L / 10 ; L / 5\}$, computed with $N=7$ 

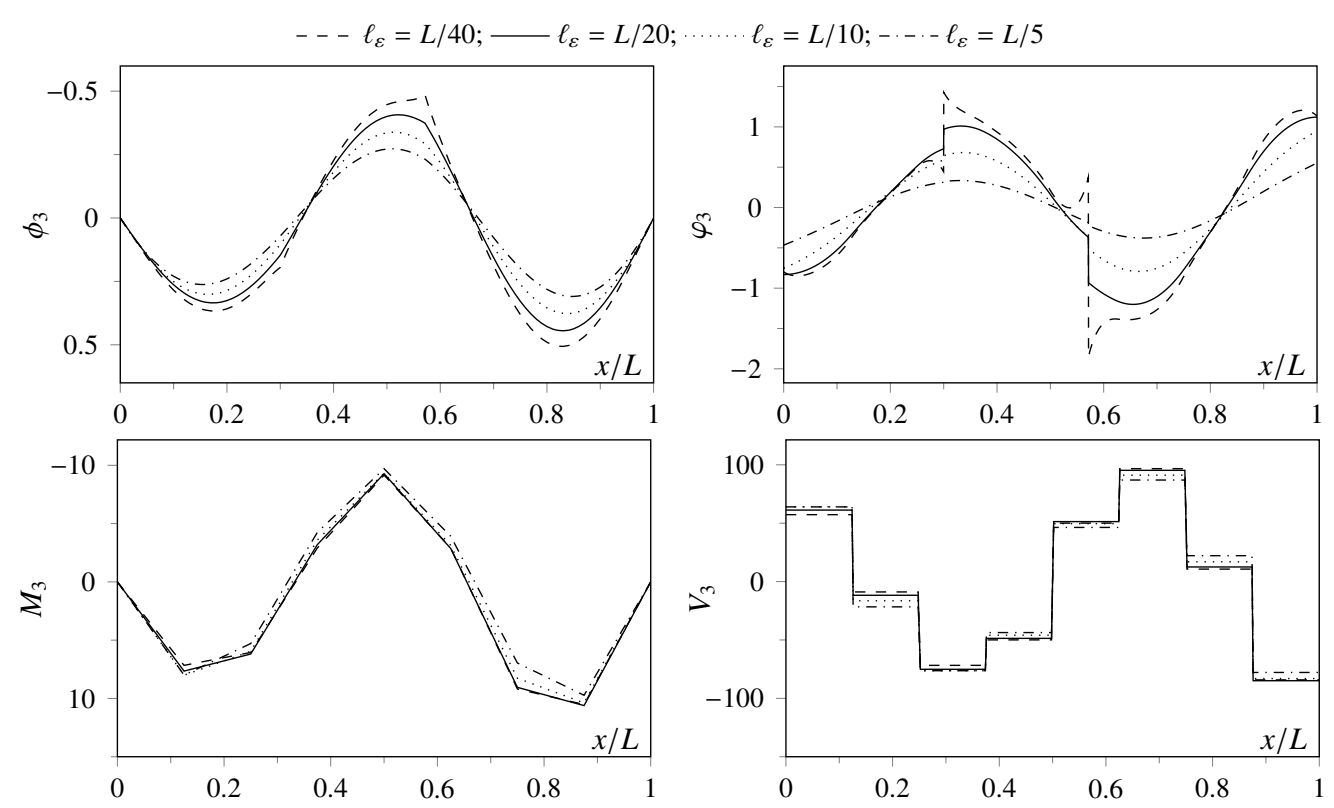

Figure 14: Example two - Deflections, rotations, shear force and bending moment diagrams of the third mode for $\ell_{\sigma}=L / 20$ and four different values of the strain length-scale parameter $\ell_{\varepsilon}=\{L / 40 ; L / 20 ; L / 10 ; L / 5\}$, computed with $N=7$
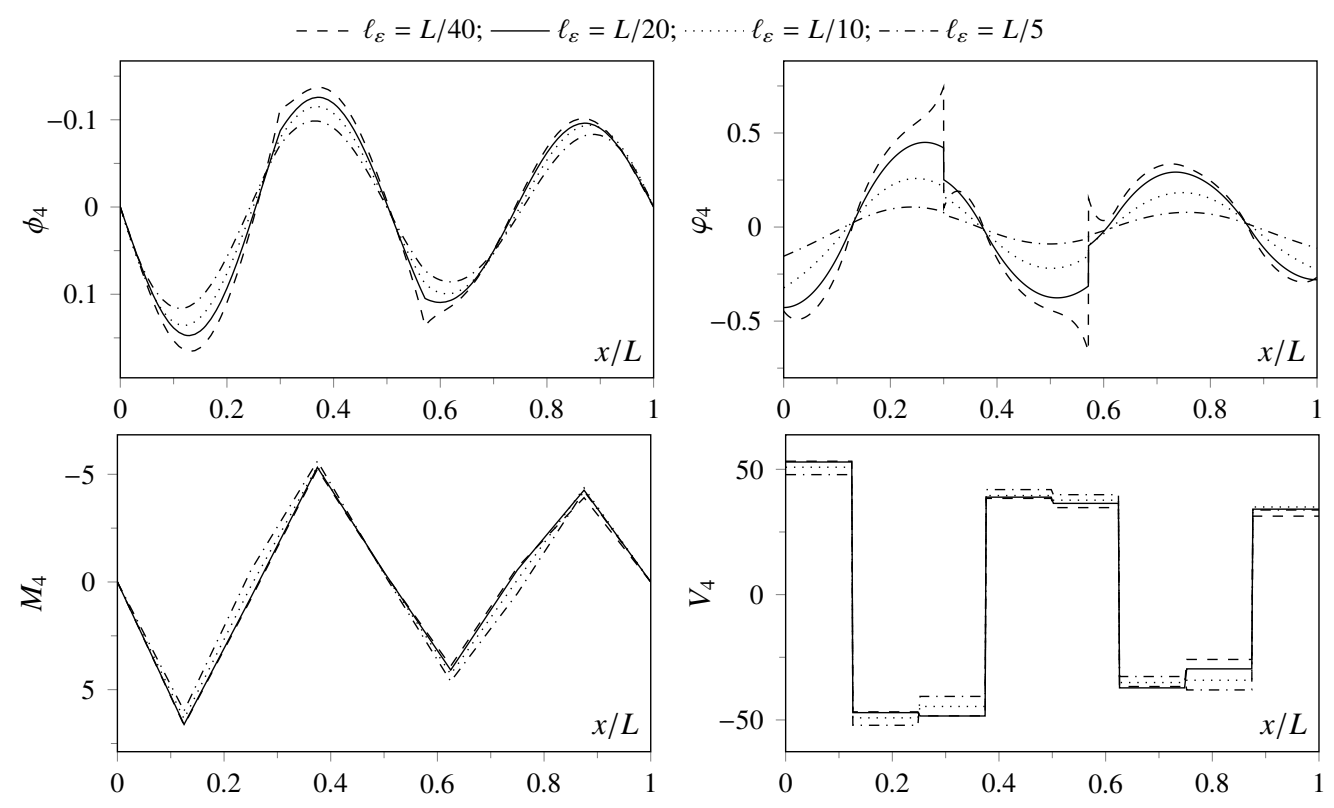

Figure 15: Example two - Deflections, rotations, shear force and bending moment diagrams of the fourth mode for $\ell_{\sigma}=L / 20$ and four different values of the strain length-scale parameter $\ell_{\varepsilon}=\{L / 40 ; L / 20 ; L / 10 ; L / 5\}$, computed with $N=7$ 


\begin{tabular}{c|c|c|c|c|c}
\hline & $\ell_{\sigma}$ & $\omega_{1}$ & $\omega_{2}$ & $\omega_{3}$ & $\omega_{4}$ \\
\hline Zhang et al. [23] & $L / 5$ & 1.382 & 4.337 & 7.808 & 11.714 \\
Proposed & $L / 5$ & $1.383(+0.07 \%)$ & $4.322(+0.09 \%)$ & $7.824(+0.20 \%)$ & $11.745(+0.26 \%)$ \\
\hline Zhang et al. [23] & $L / 10$ & 1.527 & 5.686 & 11.714 & 19.129 \\
Proposed & $L / 10$ & 1.527 & $5.690(+0.07 \%)$ & $11.729(+0.13 \%)$ & $19.165(+0.19 \%)$ \\
\hline
\end{tabular}

Table 5: First four natural frequencies $\omega_{i, \text { loc }}$ for the undamaged simply supported HGE beam with $\left\{\ell_{\varepsilon}=1 / 20\right\}$ and two different values of $\ell_{\sigma}=\{L / 5, L / 10\}$, using Zhang et al. solution and the proposed approach

\subsection{Third example - Clamped-clamped beam with three cracks}

A beam of length $L$ clamped at $x=0$ and $x=L$ is studied in the third example of Figure 16. Three cracks are present at $x_{1} / L=1 / 3, x_{2} / L=3 / 7$ and $x_{3} / L=5 / 7$, which are modelled with rotational springs with the same dimensionless parameter $\beta_{1}=\beta_{2}=\beta_{3}=0.1$. This example has been included to demonstrate the applicability of the proposed approach to study the dynamics of statically undetermined non-local beams with multiple concentrated cracks, which is the most general case for the problem in hand.

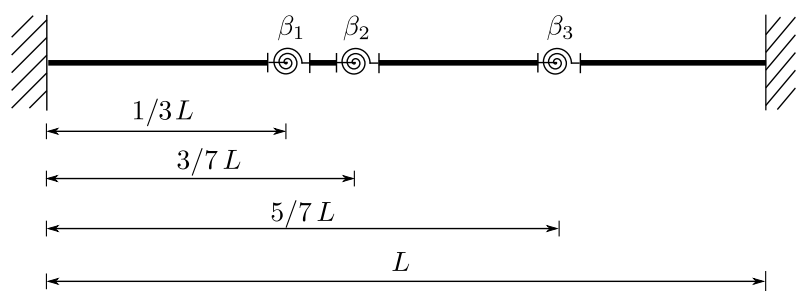

Figure 16: Example three - Clamped-clamped beam beam with three cracks

\begin{tabular}{c|c|c|c}
\hline$\omega_{1, \text { loc }}$ & $\omega_{2, \text { loc }}$ & $\omega_{3, \text { loc }}$ & $\omega_{4, \text { loc }}$ \\
\hline 3.305 & 8.440 & 17.658 & 29.725 \\
\hline
\end{tabular}

Table 6: Example three - First four natural frequencies for the local elasticity model

For illustration purposes, a relatively coarse discretisation interval $\Delta x=L / 8$ (i.e. $N=7$ ) has been chosen to plot within Figure 17 the three sets of shape functions $d^{(r)}(x), c^{(r)}(x)$ and $m^{(r)}(x)$ required by the proposed Galerkin-type approximation, along with the set of dummy point loads $P_{r}$ consecutively applied to the objective beam.

\subsubsection{Effects of the length-scale parameters}

The same type of investigations as for the previous two examples have been carried out on the beam of Figure 16, showing similar effects for the two length-scale parameters. In particular, Figures 18 and 19 display the variations of the modal frequencies with respect to the reference values of the local elasticity theory (Table 6), while Figure 20 


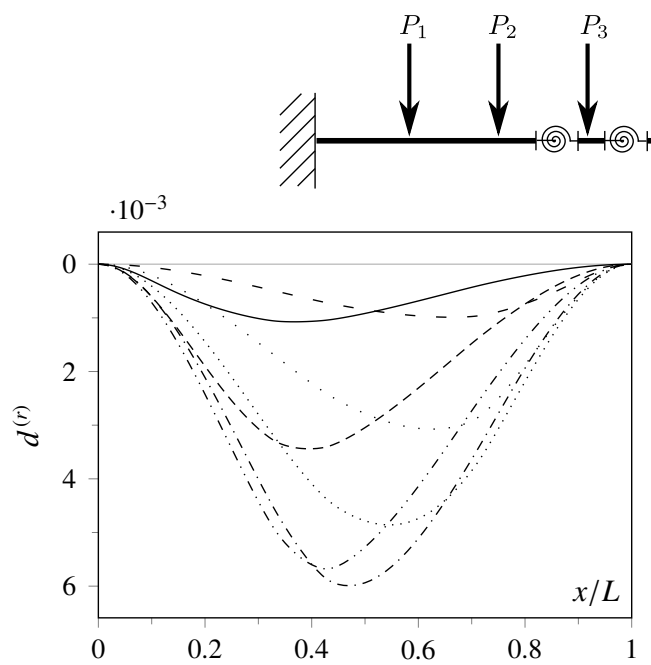

(a)

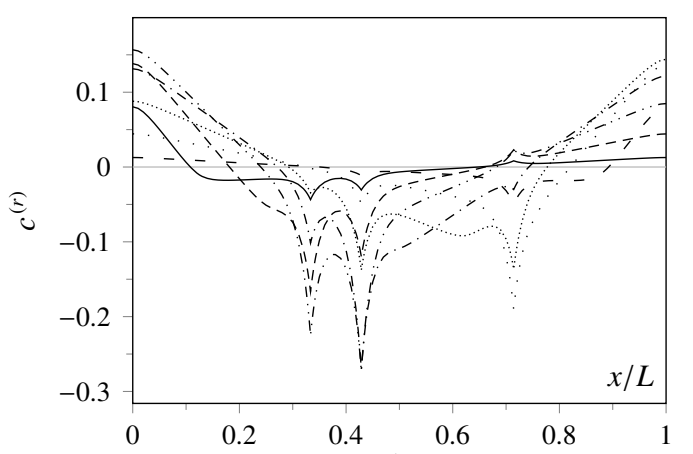

(b)

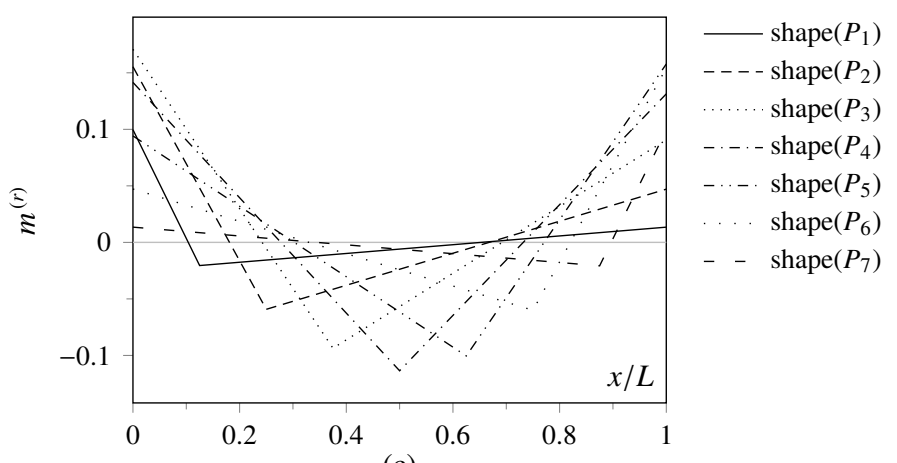

(c)

Figure 17: Example three - Transverse displacement (a), curvature (b) and bending moment (c) shape functions for $\ell_{\varepsilon}=L / 20, \ell_{\sigma}=0$ and $N=7$ 


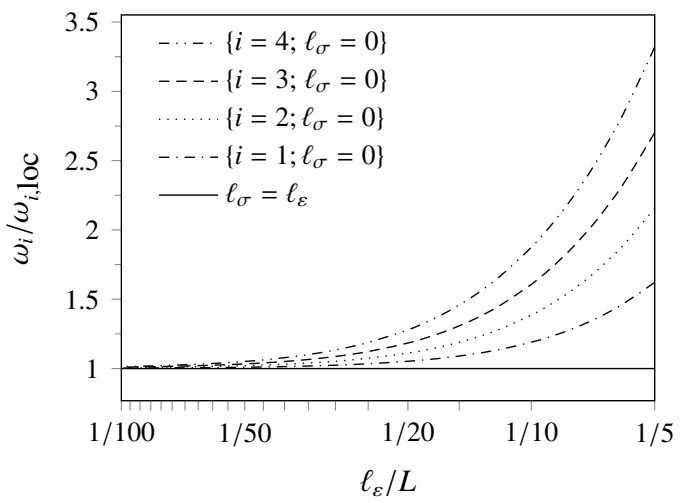

(a)

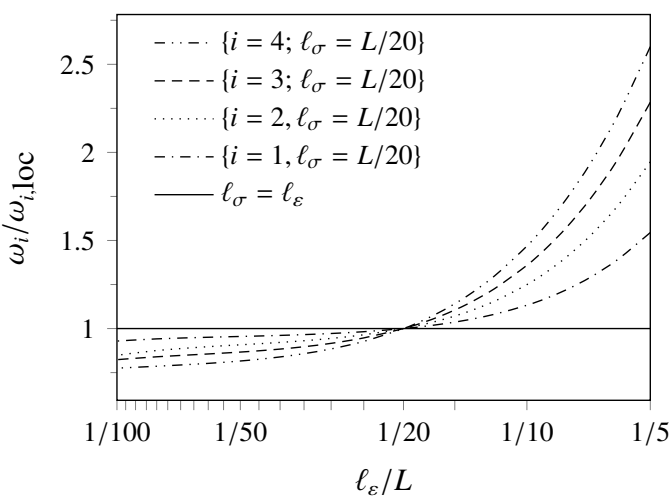

(b)

Figure 18: Example three - Normalised natural frequencies for $\ell_{\sigma}=0$ (a) and $\ell_{\sigma}=L / 20$ (b), computed with $N=15$

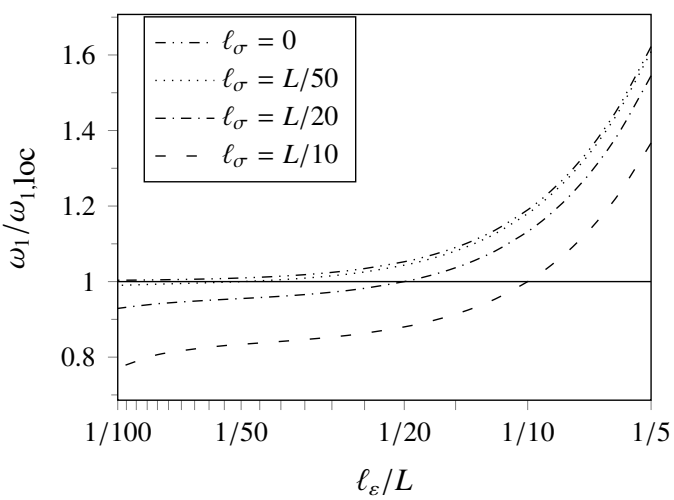

(a)

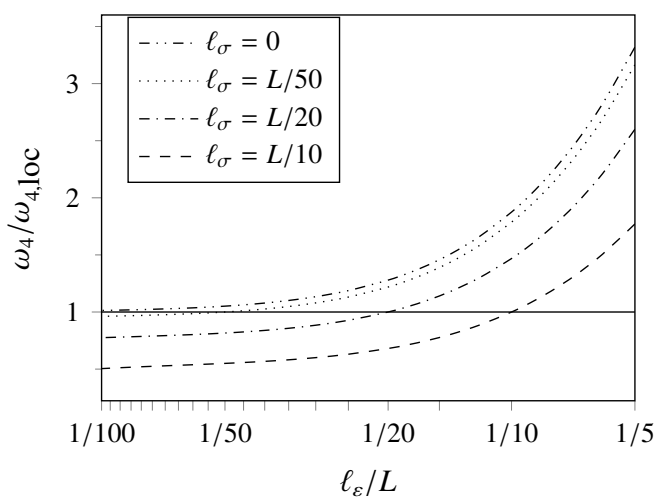

(b)

Figure 19: Example three - Normalised natural frequency $\omega_{i} / \omega_{i, \text { loc }}$ for the first (a) and fourth (b) natural frequencies of the clamped-clamped beam for several combinations of the stress length-scale $\ell_{\sigma}$ (the horizontal solid line represents the local case solution $\left.\ell_{\varepsilon}=\ell_{\sigma}\right)(N=15)$

plots the the first four modal shapes for selected microstructural parameters. As in the previous cases, it is evident the unrealistic behaviour of the cracks obtained for $\ell_{\varepsilon}<\ell_{\sigma}$ (dashed lines), while more regularised modal shapes are obtained for $\ell_{\varepsilon}>\ell_{\sigma}$ (dotted and dash-dotted lines).

\section{Concluding remarks}

In this paper, a new meshless method has been proposed for the computational dynamic analysis of multi-damaged Euler-Bernoulli (EB) beams with hybrid-gradient-elastic (HGE) constitutive law. In detail, a Galerkin-type approximation has been used, in which the shape functions are conveniently chosen as the closed-form solutions of the beam under static point loads applied at equally spaced positions along the beam. Importantly: $i$ ) the continuity of all the field variables is automatically satisfied, and there is no need to enforce any additional equilibrium/compatibility condition; ii) the same level of approximation in terms of internal forces is achieved as in the traditional finite element 

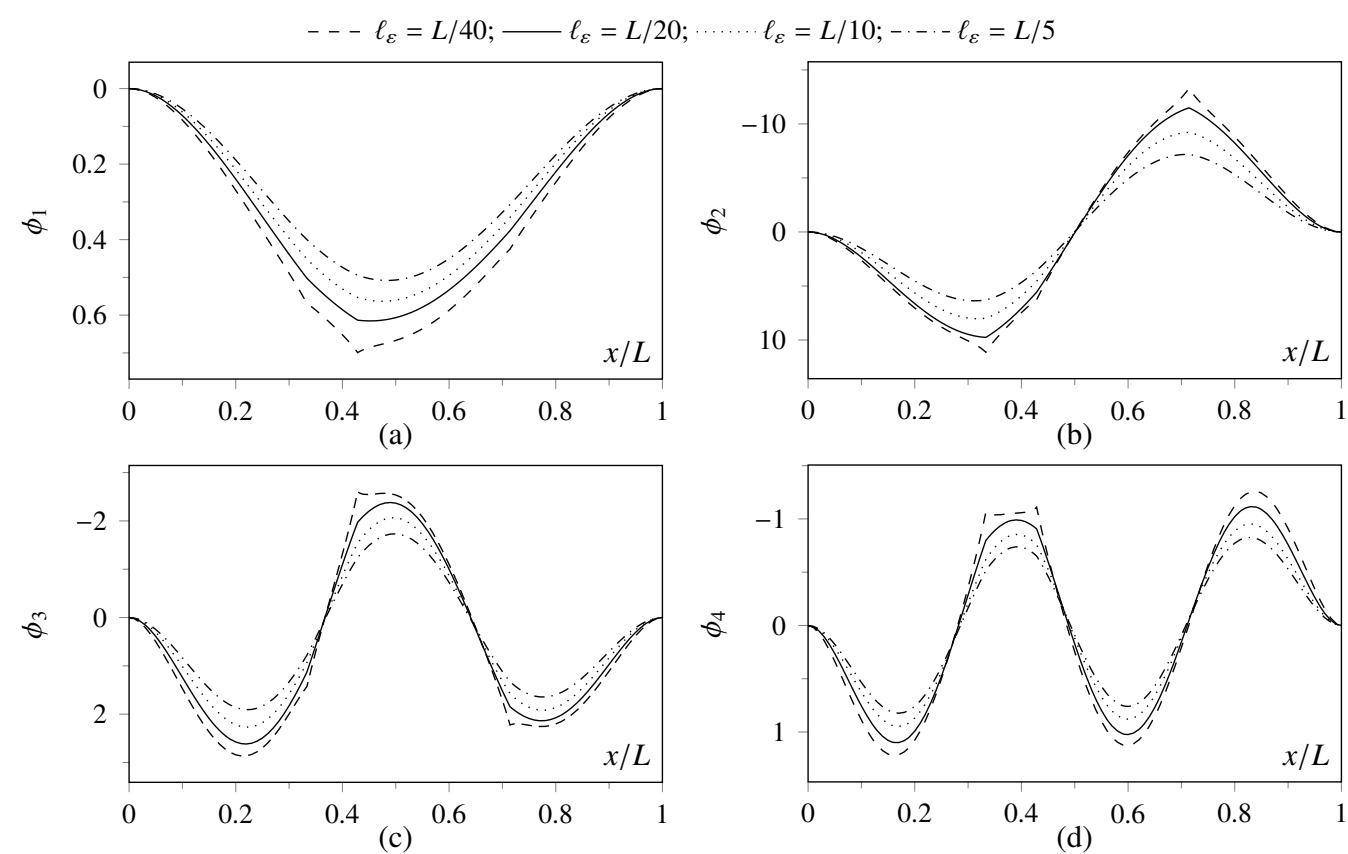

Figure 20: Example three - First four mode shapes for the clamped-clamped beam for $\ell_{\sigma}=L / 20$ and four combination of the length-scale parameters $\ell_{\varepsilon}=\{L / 40 ; L / 20 ; L / 10 ; L / 5\}(N=7)$

(FE) modelling of slender beams with classical (i.e. local) elasticity; iii) due to the adopted flexibility model, which treats the cracks as concentrated inhomogeneities, the size of the computational problem is independent of the number $n$ of cracks, and only depends on the number $N$ of shape functions being used.

The adopted HGE beam model includes two length-scale parameters for the strain $\left(\ell_{\varepsilon}\right)$ and the stress $\left(\ell_{\sigma}\right)$, which enables one to mathematically represent a variety of microstructured materials. Three numerical examples have been presented and discussed, with several combinations of the two microstructural parameters $\ell_{\varepsilon}$ and $\ell_{\sigma}$ including the reference case where $\ell_{\varepsilon}=\ell_{\sigma}>0$, which corresponds to the classical (i.e. local) elasticity theory solution.

The results have highlighted how the microstructural effects are more relevant at higher frequencies and how these effects are related to the modal shape's wave length over length-scale ratio, i.e. they start to be significant from an engineering point of view when the modal wave lengths are less than about 40 times the microstructural parameters. Furthermore, the numerical findings have confirmed that physically-consistent predictions are obtained for $0 \leq \ell_{\sigma} \leq \ell_{\varepsilon}<L$, where $L$ is the length of the beam.

\section{Appendix A. Close-form solution for the assumed shape functions}

Aim of this appendix is to provide the close-form expressions for the displacement $d^{(r)}(x)$, curvature $c^{(r)}(x)$ and bending moment $m^{(r)}(x)$ when a downward unit point load is applied at the position $x=x_{r}$, as these functions are 
needed to evaluate the stiffness and mass coefficients of the discretised beam.

The generic displacement shape function $d^{(r)}(x)$ can be expressed as the sum of an undamaged term $d_{0}^{(r)}(x)$ and the summation of the $n$ increments $d_{i}^{(r)}(x)$ due to the $n$ cracks:

$$
d^{(r)}(x)=d_{0}^{(r)}(x)+\sum_{i=1}^{n} \Delta d_{i}^{(r)}(x)
$$

where:

$$
\begin{aligned}
d_{0}^{(r)}(x) & =C_{0}^{(r)}-x C_{1}^{(r)}-\Gamma_{0}\left(\frac{x^{2} C_{2}^{(r)}}{2}+\frac{x^{3} C_{3}^{(r)}}{6}\right) \\
& -\Gamma_{0} C_{3}^{(r)} \ell_{\varepsilon}\left(\ell_{\varepsilon}^{2}-\ell_{\sigma}^{2}\right) \operatorname{sech}\left(\frac{L}{2 \ell_{\varepsilon}}\right) \sinh \left(\frac{L-2 x}{2 \ell_{\varepsilon}}\right) \\
& -2 \Gamma_{0} \ell_{\varepsilon}\left(\ell_{\varepsilon}^{2}-\ell_{\sigma}^{2}\right) \operatorname{csch}\left(\frac{L}{\ell_{\varepsilon}}\right) \cosh \left(\frac{x}{\ell_{\varepsilon}}\right) \sinh ^{2}\left(\frac{x_{r}-L}{2 \ell_{\varepsilon}}\right) \\
+ & \Gamma_{0} \frac{1}{2} H\left(x-x_{r}\right)\left\{-\frac{\left(x-x_{r}\right)^{3}}{3}+2\left(\ell_{\varepsilon}^{2}-\ell_{\sigma}^{2}\right) \times\right. \\
& {\left.\left[x_{r}-x+\sinh \left(\frac{x-x_{r}}{\ell_{\varepsilon}}\right) \ell_{\varepsilon}\right]\right\} ; } \\
\Delta d_{i}^{(r)}(x)= & -\Gamma_{0} \frac{\beta_{i}}{\ell_{\varepsilon}}\left[C_{2}^{(r)}+\bar{x}_{i} C_{3}^{(r)}+\left(\bar{x}_{i}-x_{r}\right) H\left(\bar{x}_{i}-x_{r}\right)\right] \times \\
& \left\{\operatorname{csch}\left(\frac{1}{\ell_{\varepsilon}}\right) \cosh \left(\frac{x}{\ell_{\varepsilon}}\right) \cosh \left(\frac{L-\bar{x}_{i}}{\ell_{\varepsilon}}\right)\left(\ell_{\varepsilon}^{2}-\ell_{\sigma}^{2}\right)\right. \\
& \left.+H\left(x-\bar{x}_{i}\right)\left[\ell_{\varepsilon}\left(x-\bar{x}_{i}\right)-\left(\ell_{\varepsilon}^{2}-\ell_{\sigma}^{2}\right) \sinh \left(\frac{x-\bar{x}_{i}}{\ell_{\varepsilon}}\right)\right]\right\} ;
\end{aligned}
$$

in which $C_{0}^{(r)}=d^{(r)}(0), C_{1}^{(r)}=-d^{(r)}(0), C_{2}^{(r)}=m^{(r)}(0)$ and $C_{3}^{(r)}=m^{\prime(r)}(0)$ are the four integration constants for the $r$ th point load, whose values depend on the BCs.

Analogously, the $r$ th curvature shape function $c^{(r)}(x)$ can be expressed as:

$$
c^{(r)}(x)=c_{0}^{(r)}(x)+\sum_{i=1}^{n} \Delta c_{i}^{(r)}(x),
$$


where:

$$
\begin{aligned}
c_{0}^{(r)}(x)= & \Gamma_{0}\left(C_{2}^{(r)}+C_{3}^{(r)} x\right) \\
+ & \Gamma_{0} C_{3}^{(r)} \frac{\ell_{\varepsilon}^{2}-\ell_{\sigma}^{2}}{\ell_{\varepsilon}} \operatorname{sech}\left(\frac{L}{2 \ell_{\varepsilon}}\right) \sinh \left(\frac{L-2 x}{2 \ell_{\varepsilon}}\right) \\
+ & 2 \Gamma_{0} \frac{\ell_{\varepsilon}^{2}-\ell_{\sigma}^{2}}{\ell_{\varepsilon}} \cosh \left(\frac{x}{\ell_{\varepsilon}}\right) \operatorname{csch}\left(\frac{L}{\ell_{\varepsilon}}\right) \sinh ^{2}\left(\frac{L-x_{r}}{2 \ell_{\varepsilon}}\right) \\
+ & \Gamma_{0} H\left(x-x_{r}\right)\left[x-x_{r}-\frac{\ell_{\varepsilon}^{2}-\ell_{\sigma}^{2}}{\ell_{\varepsilon}} \sinh \left(\frac{x-x_{r}}{\ell_{\varepsilon}}\right)\right] \\
\Delta c_{i}^{(r)}(x)= & \Gamma_{0} \frac{\beta_{i}}{\ell_{\varepsilon}}\left[C_{2}^{(r)}+\bar{x}_{i} C_{3}^{(r)}+\left(\bar{x}_{i}-x_{r}\right) H\left(\bar{x}_{i}-x_{r}\right)\right] \\
& \left\{\frac { \ell _ { \varepsilon } ^ { 2 } - \ell _ { \sigma } ^ { 2 } } { \ell _ { \varepsilon } ^ { 2 } } \left[\cosh \left(\frac{x}{\ell_{\varepsilon}}\right) \cosh \left(\frac{L-\bar{x}_{i}}{\ell_{\varepsilon}}\right) \operatorname{csch}\left(\frac{L}{\ell_{\varepsilon}}\right)\right.\right. \\
& \left.-H\left(x-\bar{x}_{i}\right) \sinh \left(\frac{x-\bar{x}_{i}}{\ell_{\varepsilon}}\right)\right] \\
& \left.+\frac{\ell_{\sigma}^{2}}{\ell_{\varepsilon}^{2}} \cosh \left(\frac{x-\bar{x}_{i}}{\ell_{\varepsilon}}\right) \delta\left(x-\bar{x}_{i}\right)\right\}
\end{aligned}
$$

Finally, the bending moment shape function $m^{(r)}(x)$ can simply be expressed as:

$$
m^{(r)}(x)=C_{2}^{(r)}+C_{3}^{(r)}+\left(x-x_{r}\right) H\left(x-x_{r}\right)
$$

\section{References}

[1] Donà, M., Palmeri, A., Cicirello, A., Lombardo, M.. A two-node multi-cracked beam element for static and dynamic analysis of planar frames. In: Topping, B., editor. Proceedings of the Eleventh International Conference on Computational Structures Technology. Civil-Comp Press,Stirlingshire, UK; 2012, p. 257.

[2] Caddemi, S., Caliò, I., Cannizzaro, F., Rapicavoli, D.. A novel beam finite element with singularities for the dynamic analysis of discontinuous frames. Archive of Applied Mechanics 2013;83:1451-1468.

[3] Cicirello, A., Palmeri, A.. Static analysis of Euler-Bernoulli beams with multiple unilateral cracks under combined axial and transverse loads. International Journal of Solids and Structures 2014;51:1020-1029.

[4] Donà, M., Palmeri, A., Lombardo, M.. Exact closed-form solutions for the static analysis of multi-cracked gradient-elastic beams in bending. International Journal of Solids and Structures 2014;51(15-16):2744 - 2753.

[5] Palmeri, A., Cicirello, A.. Physically-based Dirac's delta functions in the static analysis of multi-cracked Euler-Bernoulli and Timoshenko beams. International Journal of Solids and Structures 2011;48(14-15):2184 - 2195.

[6] Mindlin, R.D.. Micro-structure in linear elasticity. Archive for Rational Mechanics and Analysis 1964;16(1):51-78.

[7] Eringen, A.. On differential equations of nonlocal elasticity and solutions of screw dislocation and surface waves. Journal of Applied Physics 1983;54(9):4703-4710

[8] Eringen, A.C.. Theory of nonlocal elasticity and some applications. Tech. Rep.; DTIC Document; 1984.

[9] Eringen, A.. Nonlocal continuum field theories. Springer Verlag; 2002. 
[10] Aifantis, E.C.. On the role of gradients in the localization of deformation and fracture. International Journal of Engineering Science 1992;30(10):1279-1299.

[11] Aifantis, E.C.. Update on a class of gradient theories. Mechanics of Materials 2003;35(3):259-280.

[12] Aifantis, E.C.. On the gradient approach-relation to eringen $\varnothing \varnothing$ s nonlocal theory. International Journal of Engineering Science 2011;49(12):1367-1377.

[13] Askes, H., Aifantis, E.C.. Gradient elasticity in statics and dynamics: An overview of formulations, length scale identification procedures, finite element implementations and new results. International Journal of Solids and Structures 2011;48(13):1962-1990.

[14] Challamel, N., Girhammar, U.A.. Boundary-layer effect in composite beams with interlayer slip. Journal of Aerospace Engineering 2010;24(2):199-209.

[15] Wang, C.M., Challamel, N.. The small length scale effect for a non-local cantilever beam: a paradox solved. Nanotechnology 2008;19(34):345703.

[16] Challamel, N., Wang, C.M., Elishakoff, I.. Discrete systems behave as nonlocal structural elements: Bending, buckling and vibration analysis. European Journal of Mechanics-A/Solids 2014;44:125-135.

[17] Wang, Q., Varadan, V.K.. Vibration of carbon nanotubes studied using nonlocal continuum mechanics. Smart Materials and Structures 2006;15(2):659.

[18] Wang, C.M., Zhang, Y.Y., He, X.Q.. Vibration of nonlocal timoshenko beams. Nanotechnology 2007;18(10):105401.

[19] Murmu, T., Adhikari, S.. Nonlocal effects in the longitudinal vibration of double-nanorod systems. Physica E: Low-Dimensional Systems and Nanostructures 2010;43(1):415-422.

[20] Murmu, T., Adhikari, S.. Nonlocal transverse vibration of double-nanobeam-systems. Journal of Applied Physics 2010;108(8):083514083514.

[21] Murmu, T., Adhikari, S.. Nonlocal vibration of carbon nanotubes with attached buckyballs at tip. Mechanics Research Communications $2011 ; 38(1): 62-67$.

[22] Murmu, T., Adhikari, S., Wang, C.Y.. Torsional vibration of carbon nanotube-buckyball systems based on nonlocal elasticity theory. Physica E: Low-Dimensional Systems and Nanostructures 2011;43(6):1276-1280.

[23] Zhang, Y.Y., Wang, C.M., Challamel, N.. Bending, buckling, and vibration of micro/nanobeams by hybrid nonlocal beam model. Journal of Engineering Mechanics 2010;136(5):562-574.

[24] Loya, J., López-Puente, J., Zaera, R., Fernandez-Sáez, J.. Free transverse vibrations of cracked nanobeams using a nonlocal elasticity model. Journal of Applied Physics 2009;105(4):044309.

[25] Torabi, K., Dastgerdi, J.N.. An analytical method for free vibration analysis of Timoshenko beam theory applied to cracked nanobeams using a nonlocal elasticity model. Thin Solid Films 2012;521(21):6595-6602.

[26] Caddemi, S., Caliò, I.. Exact closed-form solution for the vibration modes of the Euler-Bernoulli beam with multiple open cracks. International Journal of Solids and Structures 2008;45:1332-1351.

[27] Mathematica, . Computer software. V.9. Http://www.wolfram.com/.

[28] Askes, H., Metrikine, A.. Higher-order continua derived from discrete media: continualisation aspects and boundary conditions. International journal of solids and structures 2005;42(1):187-202.

[29] Lombardo, M., Askes, H.. Higher-order gradient continuum modelling of periodic lattice materials. Computational Materials Science 2012;52(1):204-208.

[30] Dontsov, E.V., Tokmashev, R.D., Guzina, B.B.. A physical perspective of the length scales in gradient elasticity through the prism of wave dispersion. International Journal of Solids and Structures 2013;50(22):3674-3684. 